\title{
Quasi-dynamic Analysis, Design Optimization, and Evaluation of a Two-finger Underactuated Hand
}

\author{
M. A. Saliba ${ }^{a}$ and C. W. de Silva ${ }^{b}$ \\ ${ }^{a}$ RIAL-Robotics and Industrial Automation Laboratory, Department of Industrial and Manufacturing Engineering, University \\ of Malta, Msida, MSD 2080, Malta. E-mail: michael.saliba@um.edu.mt. Corresponding author. \\ b/ndustrial Automation Laboratory, Department of Mechanical Engineering, University of British Columbia, Vancouver, BC, \\ V6T 1Z4, Canada.E-mail: desilva@mech.ubc.ca.
}

\begin{abstract}
Underactuated hands are able to achieve shape adaptation to conformally grasp a wide variety of objects, while keeping low undesirable hand attributes such as weight, size, complexity and cost. The available analytical and simulation studies of planar underactuated hands normally assume quasi-static conditions and a fixed object. In the present paper, a new quasi-dynamic analysis of the grasping process in the horizontal plane by a planar, two-finger, four-joint underactuated hand is presented. The study considers object movement during the grasping process, and also contact friction with a surface that supports the object. An extensive and versatile simulation program, based on the analysis, is developed to investigate the effects of various parameters of hand and object on the grasping process. A prototype hand has been developed and the simulation results are validated experimentally. An extensive and detailed study and optimization exercise is carried out using the developed simulation tool. Specifically, the study concerns a manipulative grasping process that moves the object to the hand centerline during the process. Important new findings on the influence of link dimensions, link angular speeds, friction with the supporting surface, object mass and object size on the grasping performance of the hand in this scenario are presented. The results are used to establish new design guidelines for the hand. In particular, the results indicate that in the case where there is limited information on the size and precise initial location of the object to be grasped, the optimal hand design would involve inner to outer phalange size ratios of approximately $3: 1$, and inner phalange joints that are very close to each other.
\end{abstract}

Keywords - underactuated robotic hand, planar grasping, grasping dynamics, robot hand design

\section{Introduction}

An underactuated mechanism is one that has fewer actuators than degrees of freedom, and underactuated robot hands are normally suited for applications where the primary requirement is grasping rather than manipulation [1]. Such hands are able to achieve shape adaptation to a wide variety of objects, while keeping undesirable attributes such as weight, size, complexity and cost low $[1,2]$. Whereas the principle of underactuation has been applied to the design of robot hands since the 1970s [3], the classifications, analyses and other studies specific to underactuated robot hands began to appear in a systematic and consistent manner only since the late 1990 s.

Underactuated mechanisms can be classified into three main categories: the differential type (e.g., [4]) where classical mechanical elements route the motions to different links as required; the compliant type (e.g., [5]) where non-rigid elements such as springs distribute the grasping force among the fingers; and the triggered type (e.g., [6]) where clutch mechanisms are used to switch the drive between different links [7]. A basic configuration of an underactuated hand is the planar set-up shown in Fig. 1, composed of a palm and two fingers with two degrees of freedom (DOFs) per finger, driven either by one single actuator (for all four DOFs), or by two actuators (one per finger). In recent years 


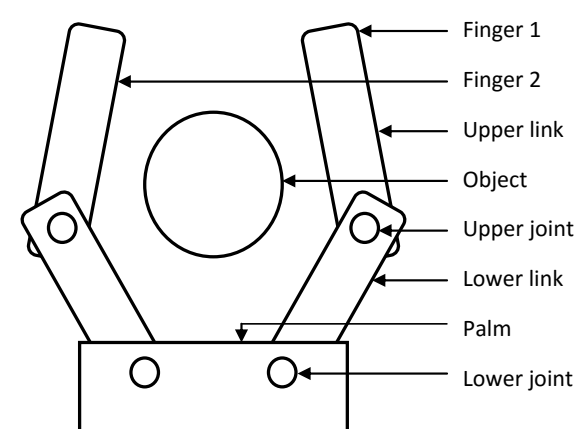

Fig. 1. Basic configuration of an underactuated hand.

several studies have been carried out on grasping an object, usually of circular cross-section, using an underactuated hand of this type [1, 8-13].

In [1] a static analysis is presented on the grasping process and a simulation program is developed to optimize a number of design parameters for the hand. The analysis assumes a fixed object, and ignores friction between the links and the object. The paper concludes that the optimum length ratio between the lower and upper phalanges of the fingers is highly dependent on the object size. A literature review of artificial hands carried out in [1] has indicated that the average ratio between the palm width and the finger length is about 0.56 . In $[8,13]$ an analysis and simulation of the grasping process using a gripper with compliant joints is presented. The behavior of the gripper is analyzed both before (i.e., during the approach and the first contact with the object) and after joint actuation. The object is assumed to be fixed. A way to distinguish between a successful enveloping grasp (defined as a situation where the object is physically constrained by the grasper against any linear displacement in the plane regardless of friction); a non-enveloping grasp (where there is no form closure however a force closure grasp is achieved through link-object friction); and an unsuccessful grasp is presented. A successful grasp range is defined as a measure of the range of initial object locations (parallel to the palm) that would result in a successful enveloping grasp, for a specific hand configuration and object size. It is concluded that, under the simulated conditions, this performance parameter is affected by the passive compliance stiffness ratio between the lower and upper links, but not by the value of the link-object friction. It is indicated that the results are more pertinent to larger object radii and therefore to actual engineering situations where the gripper size is normally optimized (minimized) to match the object size.

In $[9,12]$ the property of form closure is analyzed for an underactuated hand of the type shown in Fig. 1. It is shown that where there are backdrivable joints the conditions required for form closure are more restrictive than those for a fully actuated hand. Methods to assess the performance of underactuated hands are discussed in [10]. In particular a new metric $Q_{\text {grasp }}$ termed "ability to grasp" of the hand, given by the ratio of the size range of graspable objects $\Delta R$ and the span $S$ of the hand, is defined. This approach is demonstrated and evaluated experimentally in [11].

The literature is currently lacking in dynamic analyses of the manipulative grasping process by underactuated hands, and consequently the influence of the various hand design parameters on grasping performance is not yet clearly understood. The aim of this work is to contribute towards the filling of this research gap. The specific objectives of the present work are (i) to present a quasidynamic analysis of the manipulative grasping process by a triggered type underactuated hand with 
nonbackdrivable joints, of the basic kinematic architecture of Fig. 1; (ii) to develop very versatile simulation software based on the analysis; (iii) to perform experimental evaluation to validate the model; (iv) to use the validated model to optimize design parameters for the case of a self-centering manipulative grasp where there is limited information on the size and on the precise initial location of the object to be grasped; (v) to summarize the results in a design approach for this type of hand; and (vi) to discuss the applicability and limitations of the results. It is noted that consideration of a triggered type hand with nonbackdrivable joints provides for a more basic dynamic analysis - the results can later be extended to other types of underactuated hands and of joints as indicated in section 8.

This work is very relevant to many real world scenarios. For example as part of a flexible automated assembly operation in industry that involves objects of various sizes, a planar underactuated hand may be required to grasp the object (that initially rests on a conveyor) for relocation purposes, in a manner that is robust to errors in the initial position of the object, but that results in a grasp where the object is centred about the line of symmetry of the hand for correct placement after relocation. Alternatively, a domestic robot may need to have the capability to grasp objects of various sizes that lie on horizontal surfaces, without the need to employ high positional accuracy during approach, and to automatically centre the object within the grasp in order to simplify the relocation task.

This paper is organized as follows: Section 1 has introduced the topic, identified the research gap, and outlined the objectives and motivation of this work. Section 2 presents a detailed quasi-dynamic analysis of the manipulative grasping process in terms of various hand and object variables, including palm and link dimensions, joint velocities, threshold torques, object size, weight and initial position, friction between the object and the links, and friction between the object and the supporting surface. Section 3 describes the versatile simulation program that was developed based on this analysis. Section 4 describes the experimental validation of the simulation program. Section 5 discusses issues pertaining to parameter normalization; defines the performance metrics; and outlines the general conditions that were applied during the simulation runs. Section 6 investigates in detail the effects of various parameters of the underactuated planar gripper on the successful grasp range and grasping ability, and applies the results obtained towards the suggestion of an optimized hand design for the case where there is limited information on the size and precise initial location of the object to be grasped, and where it is important that the grasp is one that envelops the object. Section 7 extends the discussion to summarize and rationalize the results obtained and their applicability. Finally, section 8 summarizes the contribution of the paper and suggests future work that can build on these results.

\section{Analysis of the grasping process}

\subsection{Problem formulation}

The general grasping process that is analyzed is the following: The underactuated gripper is of the basic architecture of Fig. 1 and is of the triggered type with nonbackdrivable joints. The object lies on a flat horizontal surface and is allowed to move during grasping. Each of the two fingers of the gripper is driven by a single actuator. The actuation is first imparted to the lower joint of the finger. When the resisting torque of the lower joint reaches a threshold value, the actuator drive switches over to the upper joint. The upper link continues to close until the resisting torque of the upper joint reaches a preset limit. 
A typical robotic grasping process of a two-fingered four-link gripper for an arbitrarily shaped object is shown in Fig. 2. The lower links rotate towards the object until contact is made by one of the fingers, characteristically (but not necessarily) by the lower link (Fig. 2(a)). If the torque limit for the lower joint of the contacting finger is not reached, the lower link continues to rotate, moving the object across the supporting surface, until contact with the lower link of the second finger is made (Fig. 2(b)). At this point the threshold torque for both lower joints will likely be reached, in which case the drives switch over to the upper joints. The upper links continue to rotate until the torque limits for the upper joints are reached due to contact with the object (Figs 2(c) and 2(d)).

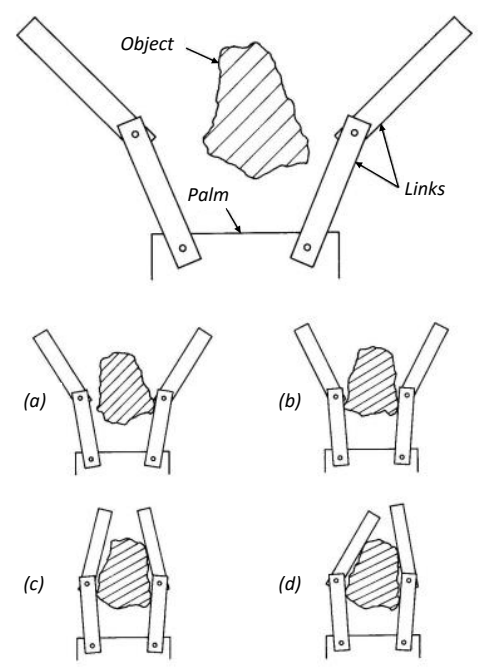

Fig. 2. A typical grasping process with a two-link two-finger hand.

As in the previous work, the analysis is simplified by assuming an object of circular cross-section, in the present case a disk of radius $R$, resting with one of its flat sides in contact with the supporting surface. The parameters that are pertinent to the analysis are illustrated in Figure 3 and/or described in Table 1.

The analysis is based on several assumptions that serve to simplify the analysis. First, the object and the gripper links are assumed rigid. Second, the actuators are assumed to serve as sources of constant velocity. Third, contact bounce is neglected. Fourth, as a consequence of the first and the second assumptions, the short period of initial acceleration from rest of the object is neglected, giving it instantaneous non-zero linear and rotational velocities on initial contact with the link, in order to avoid interpenetration of the object and the links and to satisfy the ensuing equations of motion. Similarly, object inertia and deceleration during the impact interval when an object being pushed by one finger contacts the second finger, is neglected. It is noted that the angular speeds of the links are sufficiently small to justify these four assumptions. In the practical application, a compromise between the pair of assumptions (i) and (ii) (which would both tend to promote contact bounce) and assumption (iii) is reached by using thin rubber pads on the tactile surfaces. These pads, as well as friction between the object and the supporting surface will tend to dampen the impact response characteristics, serving to eliminate contact bounce without being expected to cause severe deviation from the approximation made under the first two assumptions above. A prototype gripper that was used to validate the analysis and simulation was driven by stepper motors [14], and as such the second assumption is valid 
during steady state motion if no motor steps are missed. For a gripper that is driven by DC servo motors [14], it is assumed that the gripper motion prior to grasp attainment will be sufficiently overpowered to justify this second assumption. The fifth assumption made in the analysis is that of Coulomb friction model between the object and the links, and between the object and the supporting surface.

\subsection{One-link contact analysis}

The entire analysis of the present subsection is based on the situation where there is contact only with the lower link of finger 1 , denoted as Link $A$ in Fig. 3 . The equations for one-link contact with Links $B$, $C$, and $D$ can be derived in a similar manner to Link $A$.

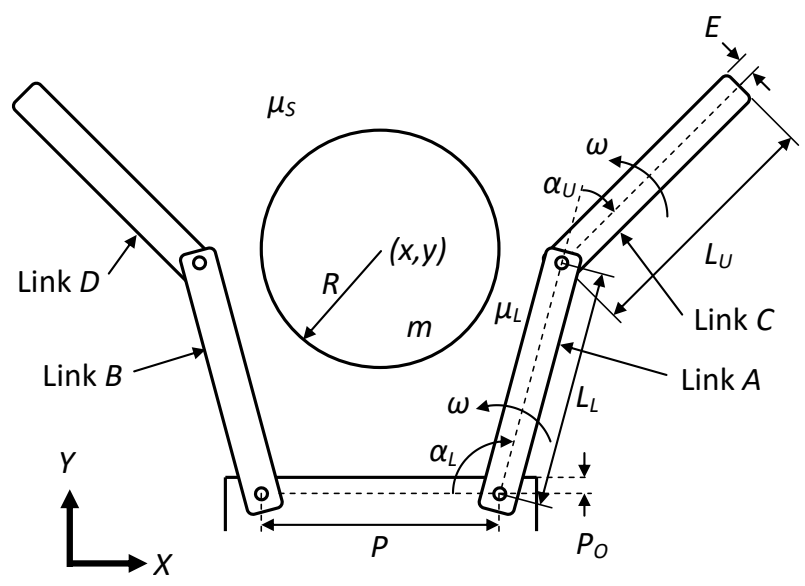

Fig. 3. Parameters of the grasping process.

\subsubsection{Kinematics of object motion}

With reference to Fig. 4(a) the following kinematic equations are written:

$$
\begin{gathered}
\boldsymbol{p}=\boldsymbol{\ell}_{\boldsymbol{A}}+\boldsymbol{E}+\boldsymbol{R}=\ell_{A} \boldsymbol{i}_{\boldsymbol{\ell}}+(R+E) \boldsymbol{i}_{\boldsymbol{\alpha}} \\
\dot{\boldsymbol{p}} \equiv \boldsymbol{v}=\left[\dot{\ell}_{A}-\omega_{A}(R+E)\right] \boldsymbol{i}_{\boldsymbol{\ell}}+\left[\omega_{A} \ell_{A}\right] \boldsymbol{i}_{\boldsymbol{\alpha}} \\
\ddot{\boldsymbol{p}} \equiv \dot{\boldsymbol{v}}=\left[\ddot{\ell}_{A}-\omega_{A}^{2} \ell_{A}\right] \boldsymbol{i}_{\boldsymbol{\ell}}+\left[2 \omega_{A} \dot{\ell}_{A}-(R+E) \omega_{A}^{2}\right] \boldsymbol{i}_{\boldsymbol{\alpha}}
\end{gathered}
$$

From Eq. (2) one has,

$$
\psi_{A}=\tan ^{-1} \frac{\dot{\ell}_{A}-\omega_{A}(R+E)}{\omega_{A} \ell_{A}}
$$

\subsubsection{Case 1: No slip at object-link contact point}

With reference to Fig. 4(b) and using the results of Eq. (3), the translational dynamics in the $\boldsymbol{i}_{\boldsymbol{\alpha}}$ direction give

$$
F_{N A}-F_{f} \cos \psi_{A}=2 m \omega_{A} \dot{\ell}_{A}-m \omega_{A}^{2}(R+E)
$$


The rotational dynamics give

$$
\left(m \omega_{A}^{2} \ell_{A}-m \ddot{\ell}_{A}\right) R-T_{f}-\left(F_{f} \sin \psi_{A}\right) R=-\frac{m R^{2}}{2} \ddot{\theta}
$$

Since there is no slip between the object and the link, one has

$$
\ddot{\ell}_{A}=-R \ddot{\theta}
$$

Substituting Eq. (7) into Eq. (6) one obtains

$$
\left(m \omega_{A}^{2} \ell_{A}\right) R-T_{f}-\left(F_{f} \sin \psi_{A}\right) R=-\frac{3 m R^{2}}{2} \ddot{\theta}
$$

With reference to Fig. 4(c), it is seen that the point of contact between the link and the object has a velocity $\boldsymbol{V}_{\boldsymbol{C}}$. Thus, for the no slip condition, the object has the following initial velocities at the instant of first contact:

$$
\dot{\theta}(0)=\frac{-V_{R}}{R}=\frac{-V_{C} \sin \varepsilon}{R}
$$

\begin{tabular}{|c|c|c|}
\hline Symbol & Description & Fig. \\
\hline$P$ & Palm width & 3 \\
\hline$P_{O}$ & Palm offset distance & 3 \\
\hline$L_{L}$ & Length of lower links & 3 \\
\hline$L_{U}$ & Length of upper links & 3 \\
\hline$E$ & Link offset distance & 3 \\
\hline$\omega$ & Generalized joint speeds & 3 \\
\hline$\alpha_{L}$ & Generalized lower link angles & 3 \\
\hline$\alpha_{U}$ & Generalized upper link angles & 3 \\
\hline$\mu_{L}$ & Friction coefficient object-link & 3 \\
\hline$\mu_{S}$ & Friction coefficient object-surface & 3 \\
\hline$R$ & Object radius & 3 \\
\hline$m$ & Object mass & 3 \\
\hline$\alpha_{A}$ & Joint position for Link $A$ & 4(a) \\
\hline$\omega_{A}$ & Joint speed for Link $A$ & $4(a)$ \\
\hline$p$ & Position vector of object centroid & 4(a) \\
\hline$\ell_{A}$ & Distance of contact point up Link $A$ & 4(a) \\
\hline$\theta$ & Angular position of object & $4(a)$ \\
\hline$v$ & Linear velocity vector of object centroid & $4(a)$ \\
\hline$\psi_{A}$ & Direction of $\boldsymbol{v}$ wrt contact normal at Link $A$ & $4(a)$ \\
\hline$F_{N A}$ & Normal contact force at $\operatorname{Link} A$ & $4(\mathrm{~b})$ \\
\hline$F_{f}$ & Friction force at object center, due surface & $4(\mathrm{~b})$ \\
\hline$T_{f}$ & Friction torque at object center, due surface & $4(b)$ \\
\hline$L_{A}$ & Position vector of contact point & 4(c) \\
\hline$\varepsilon$ & Angle between $\boldsymbol{L}_{\boldsymbol{A}}$ and $\boldsymbol{\ell}_{\boldsymbol{A}}$ & $4(c)$ \\
\hline$V_{C}$ & Velocity vector of contact point & $4(c)$ \\
\hline$\Psi$ & Angle subtended by two contacting links & $5(a)$ \\
\hline $\boldsymbol{F}_{N B}$ & Normal contact force at Link $B$ & $5(a)$ \\
\hline$\alpha_{B}$ & Joint position for Link $B$ & $5(b)$ \\
\hline$\omega_{B}$ & Joint speed for Link $B$ & $5(b)$ \\
\hline$\varphi$ & Direction of $\boldsymbol{v}$ wrt orientation of palm & $5(b)$ \\
\hline$T$ & Generalized joint torque thresholds/limits & \\
\hline$t$ & Time & \\
\hline
\end{tabular}

$\boldsymbol{v}(0)=V_{R} \boldsymbol{i}_{\boldsymbol{\ell}}+V_{N} \boldsymbol{i}_{\boldsymbol{\alpha}}=\left[V_{C} \sin \varepsilon\right] \boldsymbol{i}_{\boldsymbol{\ell}}+\left[V_{C} \cos \varepsilon\right] \boldsymbol{i}_{\boldsymbol{\alpha}}$

Table 1 - Nomenclature for the dynamic analysis (section 2). 


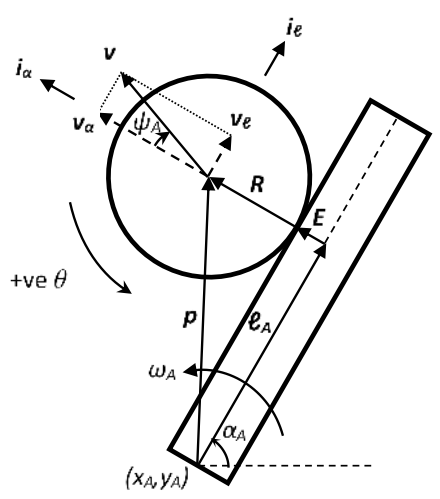

(a)

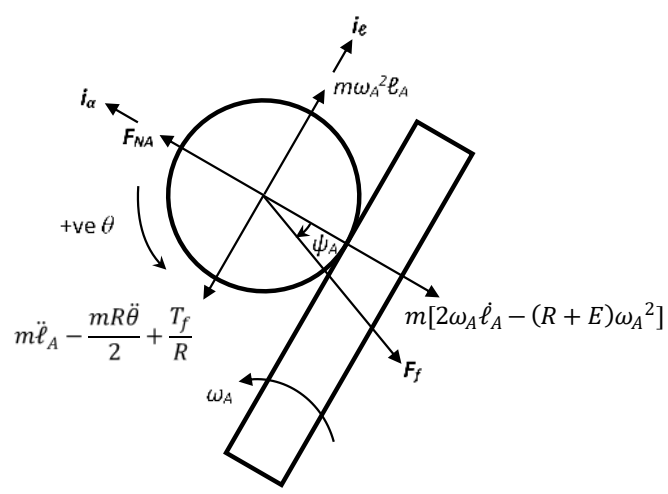

(b)

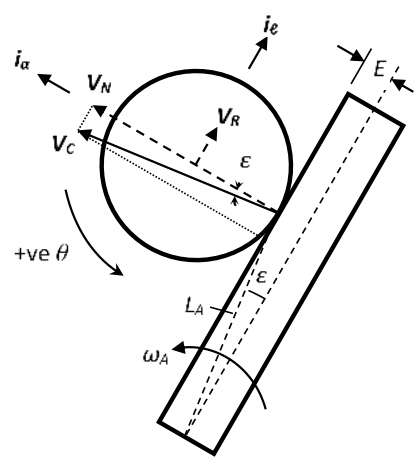

(c)

Fig. 4. One-link contact analysis. (a) link and object positions and velocities, (b) forces acting on the object, (c) contact point velocity.

where $V_{C}=\omega_{A} L_{A} ; L_{A}=\sqrt{\ell_{A}^{2}+E^{2}}$; and $\varepsilon=\tan ^{-1} \frac{E}{\ell_{A}}$. It is emphasized that the link offset $E$ has a significant effect on the object motion during one-link contact, and is not negligible. The radial component $V_{R}$ of the contact point velocity is the major factor that determines the rolling velocity of the object up the link when there is no link-object slip, and is highly dependent on the value of $\varepsilon$ and therefore of $E$.

\subsubsection{Case 2: Slip at object-link contact point}

This situation will occur if the friction at the object-link contact point is unable to provide sufficient torque to rotationally accelerate the object by the amount calculated in Eq. (8) (denoted $\ddot{\theta}^{\prime}$ below to indicate that this is a virtual value), i.e. if

$$
\left(\mu_{L} F_{N A}\right) R<-\frac{m R^{2}}{2} \ddot{\theta}^{\prime}+T_{f}
$$

When there is slip at the link, $\ell_{A}$ and $\theta$ become independent of each other. The tangential contact force on the object becomes a function of $F_{N A}$ only, and is equal to $\mu_{L} F_{N A}$. Eq. (5) is still valid, and the translational dynamics in the $\boldsymbol{i}_{\boldsymbol{\ell}}$ direction give

$$
m \omega_{A}^{2} \ell_{A}-F_{f} \sin \psi_{A}-\mu_{L} F_{N A}=m \ddot{\ell}_{A}
$$

The initial angular velocity of the object on contact is zero, and the angular acceleration is now given by the new rotational dynamics equation:

$$
\left(\mu_{L} F_{N A}\right) R-T_{f}=-\frac{m R^{2}}{2} \ddot{\theta}
$$

\subsection{Two-link contact analysis}

\subsubsection{Graspability}

As indicated in [15], a two-finger grasp is in equilibrium if 
where $\Psi$ is the angle between the two contact normals and $\lambda$ is the friction angle at the contact points. In the present study, the condition (14) is used to test for equilibrium of two-link contact situations. An additional force, the frictional force exerted by the supporting surface, is also taken into consideration since it contributes towards equilibrium of the grasp. The forces acting when the condition (14) is not satisfied, for contact with the two lower links of the gripper, are shown in Fig. $5(\mathrm{a})$. By symmetry and under the stated assumptions, one has $F_{N A}=F_{N B}$. Thus, the object will not slide out of the grasp if

$$
\mu_{S} m g \geq 2 F_{N A}\left(\sin \frac{\Psi}{2}-\mu_{L} \cos \frac{\Psi}{2}\right)
$$

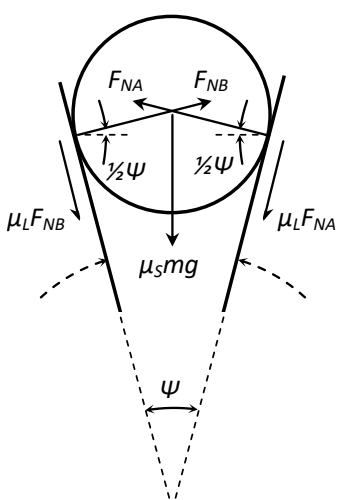

(a)

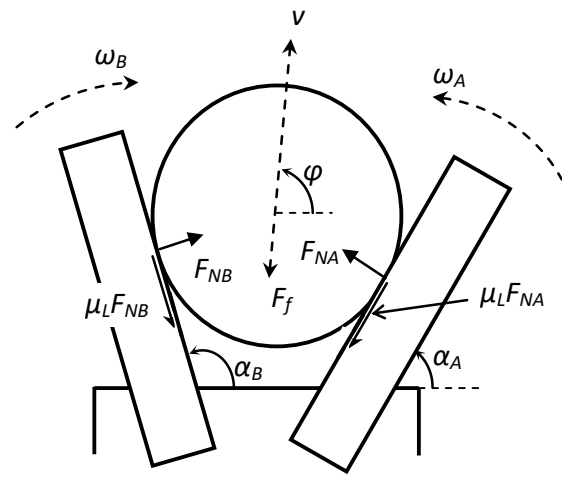

(b)

Fig. 5. Two-link contact analysis: (a) forces acting at the onset of slip, (b) Configuration 1 grasp without equilibrium.

\subsubsection{Object slip}

If both conditions (14) and (15) are not met, the object will tend to slide outwards along the links. Since the contact forces and the friction coefficients are taken to be equal on both links, it is assumed that there would be slip of the object at both links, and hence that there would be no rotation of the object during its motion along the links.

A distinction is made between three possible configurations of two-finger grasps. A Configuration 1 grasp is one that tends to squeeze the object out of the grasp. For a two-link contact with the lower links, this is defined by $\alpha_{B}>\alpha_{A}$, and is shown in Fig. $5(\mathrm{~b})$. Since the links move with constant angular velocities, the position, velocity and acceleration of the object are geometrically defined if contact with the two links is to be maintained, and are functions of the variables $\alpha_{A}, \alpha_{B}, \omega_{A}$ and $\omega_{B}$ only. The equation of motion of the object centroid is given by

$$
F_{N A} \sin \left(\varphi-\alpha_{A}\right)+F_{N B} \sin \left(\alpha_{B}-\varphi\right)-\mu_{L} F_{N A} \cos \left(\varphi-\alpha_{A}\right)-\mu_{L} F_{N B} \cos \left(\alpha_{B}-\varphi\right)=m \dot{v}+F_{f}
$$

where $F_{N A}=F_{N B}$. In the analysis of this situation, the following is taken into consideration: If the value of $F_{N A}$ (and $F_{N B}$ ) that is required to satisfy Eq. (16) under the geometric constraints of $\dot{v}$ would cause the threshold torques of either Link $A$ or Link $B$ to be exceeded, the motor drive(s) switches over to the upper link(s), and if both drives switch over the object will not slip. 
A Configuration 2 grasp, defined by $\alpha_{B}<\alpha_{A}$, is one that tends to squeeze the object into the grasp, and can be analyzed in a manner similar to the above. A Configuration 3 grasp, defined by $\alpha_{B}=\alpha_{A}$, refers to the critical case where the object does not slip due to the co-linearity of $F_{N A}$ and $F_{N B}$. The entire two-contact analysis for other combinations of link pairs (i.e., other than the pair made up of Link $A$ and Link $B$ ) can be carried out in a similar manner.

\subsection{Three- and four-link contact analyses}

\subsubsection{Form closure check}

All the gripper joints are assumed to be nonbackdrivable. Hence a grasp with three or more links has translational form closure in the plane if all adjacent pairs of normal contact forces are at an angle less than $\pi$ to each other, measured on the side of their adjacency. The "translational" descriptor is used because a circular object can never have full form closure since an external moment about its center cannot be geometrically constrained. In this paper the terminology of [8] is used, and a grasp with translational form closure is referred to as a successful enveloping grasp.

\subsubsection{Grasp equilibrium check}

If there is no translational form closure in a grasp where three or more links are involved, then the pair of adjacent links for which the normals subtend an angle greater than $\pi$ are termed the "outer" links. An equilibrium check similar to that described in subsection 2.3.1 can be applied to these links. For a grasp that is in equilibrium, the normal contact forces at the two "outer" link contact points are equal, whereas the contact forces at all the other links are zero.

\section{Simulation of the grasping process}

A comprehensive simulation program has been developed based on the analysis of section 2 , in order to investigate the grasping process by a planar underactuated gripper of the present type. The software has been developed in FORTRAN 77, in order to enable the use and extension of a large number of dedicated subroutines that were originally developed and extensively scrutinized and debugged at the University of British Columbia in the 1990s. $[2,16]$ It was considered that the drawbacks associated with the use of this programming language were well mitigated by the benefits to be gained by using and extending an available versatile resource that had already been rigorously developed and tested over a period of more than one year in the solution of the complex problem at hand. The recent additions to the code, that have been introduced to carry out the optimization exercises reported in section 6 of this work, have been subjected to the same high degree of scrutiny, and all of the results obtained (including some that were originally quite unexpected) can be well understood and explained in retrospect.

A simplified representation of the program structure is shown in Fig. 6. The input data are read from a number of input files, and these data are validated during program initialization. The program then evaluates a five bit variable "CONTACT," which holds the information on which of the four links (and/or the palm) are in contact with the object at any time during program execution. It directs the program to the appropriate subroutines to simulate the current stage of the grasping process. Next, a time increment is applied, and this is followed by the execution of the applicable dynamic and static subroutines to determine the evolution of the grasp during the time increment and to calculate/update all of the relevant variables pertaining to the grasp. During the execution of these 
routines, the program continually writes data to a number of output data files. The program then checks whether the object is still within the workspace of the gripper, and if not the program terminates. Next, the program checks whether at least one motor is still on, in which case program control is returned to the section that (re)-evaluates CONTACT. If both motors are turned off, the program terminates.

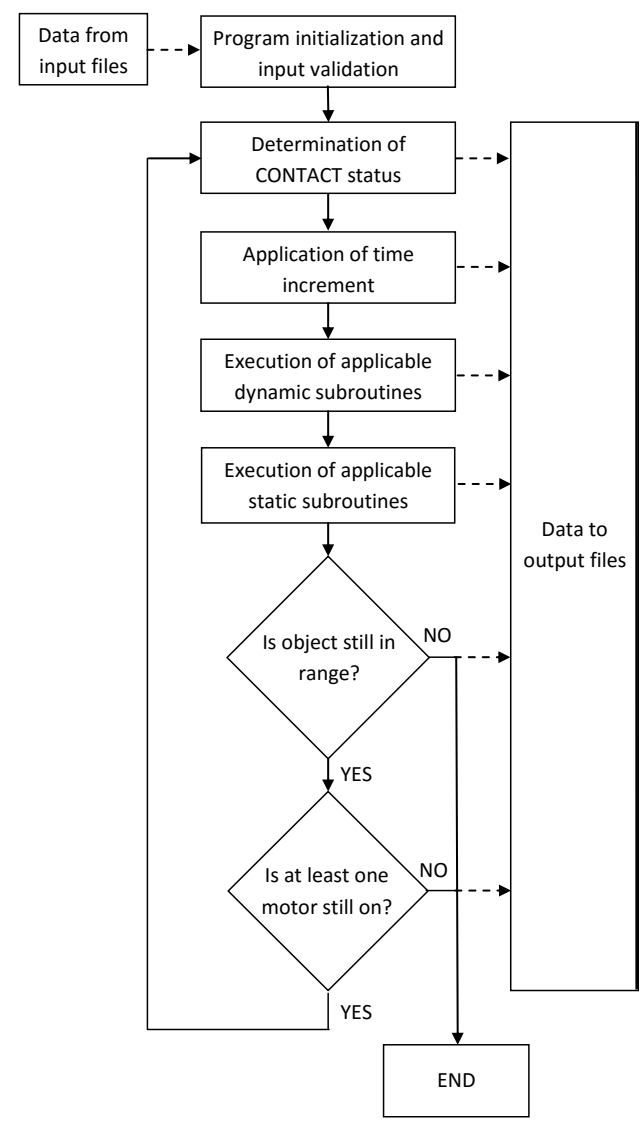

Fig. 6. General structure of the simulation program.

The program subroutines are categorized into four types. The first type corresponds to the analyticalstatic (A-S) routines, where the analysis of a situation is carried out; however, there is no link actuation or object movement. An example of an A-S routine is the one that checks whether a three-link grasp composed of contact with Links $A, B$ and $D$ has form closure, grasp equilibrium without form closure, or lack of equilibrium. The second type corresponds to the analytical-dynamic $(A-D)$ routines, which simulate the dynamic behavior of the object, and involve the solution of the dynamic equations of motion through numerical simulation. An example of an A-D routine is the one that simulates pushing and rolling of the object during contact with Link $A$. The third type corresponds to the geometricdynamic $(G-D)$ routines, which involve link or object movements without the need to solve dynamic equations of motion. An example of a G-D routine is the one that simulates the actuation of Link C when there is no contact of this link with the object. The fourth type corresponds to the geometricstatic (G-S) routines, which are responsible for making static geometric calculations of variables for use by other subroutines or by the main program. An example of a G-S routine is the one that checks whether Link $D$ has made contact with the object. 
The program is written in a manner that allows a very wide versatility of simulation. The parameters of the hand that can be varied include the palm width, the lower link lengths, the upper link lengths, the link and palm offsets, initial joint angles for each of the four links, the joint velocities, and the torque thresholds/limits of all joints. The object parameters that can be varied include the radius, the initial position ( $x$ and $y$ ), the mass, the friction with the supporting surface, and the friction with the gripper links. The overall integration time increment for the program can also be varied. In the present simulation study this was set to $\delta t=0.001 \mathrm{~s}$, since it was found that all results converged satisfactorily at this value. The output of the program consists of files that record the evolution of all the parameters that change with time, a log of the sequence of routines accessed by the program, and an assessment of whether the outcome is a successful enveloping grasp, a non-enveloping (but still in equilibrium) grasp, or a failed grasp.

A distinction between static and dynamic friction coefficients was made throughout the program as appropriate, with the latter values set to 0.75 times the former. Furthermore, it was recognized that the simultaneous translational and rotational motion of the object on the supporting surface is subject to the Contensou effect $[17,18]$, under which the frictional forces and torques become less than their maximum values. A model for this effect based on numerical integration was developed by the authors, and the model results were applied to the ongoing determination of $F_{f}$ and $T_{f}$ as functions of $v$ and $\dot{\theta}$ during the simulation.

\section{Experimental verification}

Prior to the employment of the simulation tool towards the extensive optimization task for which it had been developed, it was crucial to obtain experimental validation of its fidelity. This was done by comparing results obtained using the simulation to corresponding results obtained using a physical model.

To this end, a prototype gripper of the type described in Fig. 1 and section 2.1, and shown in Fig. 7 [2], has been developed. The simulation routines that lead to equilibrium grasps, and the associated analysis, have been assessed by means of a series of experiments carried out using this prototype. Solid cylindrical aluminum test objects of different diameters and/or weights were manufactured and used in the testing. The static friction coefficient between the aluminum objects and the (Perspex) supporting surface was determined experimentally by finding the angle of inclination of the surface at which an object, placed on the surface, would start to slide under the influence of its own weight. The value of $\mu_{S}$, averaged over more than 40 readings using five objects, was found to be 0.31 , with individual readings varying between 0.22 and 0.39 .

The experimental set-up used for the grasping tests is illustrated in Fig. 8. The closing of the gripper, as it grasped the object, was video recorded using an overhead mounted CCD camera. A stopwatch placed beside the gripper provided an accurate measure of time. The videos of the grasping processes were subsequently analyzed, and the trajectory of the object centroid and the change in object angular position during the evolution of the grasp were measured. Each grasping experiment was run six times in order to confirm repeatability of the experimentation and in order to extract an average value for object angular displacement.

A specimen result from an experimental run, and its comparison to the corresponding simulated result, are shown in Fig. 9. It was found that the simulation program consistently tended to return 
marginally higher values for the final angular displacement of the object than did the corresponding experiments. This discrepancy may be attributed to the assumptions made in the analysis (and reflected in the simulation) and to experimental error. In the simulation, in particular, the discrete angular velocity change assigned to the object on first contact by a link and the assumption of ideal Coulomb friction behavior (at the link and also at the supporting surface), both tend to contribute to an overestimation of the total angular displacement of the object during grasping. The main sources of experimental error are resolution limitations in the measurement of object angular position and mechanical limitations of the prototype gripper (e.g., occasional non-consistent motor drive switchover thresholds, and backlash in the gearing and cable transmissions).

The coefficient of determination (as a measure of goodness of fit) of the experimental data to the simulated curves was consistently above 0.985 for the trajectory plots and 0.99 for the angular position plots. The experimental results indicate that the simulation program is a reliable tool that can be used for a detailed investigation of the grasping process by a gripper of the present type.

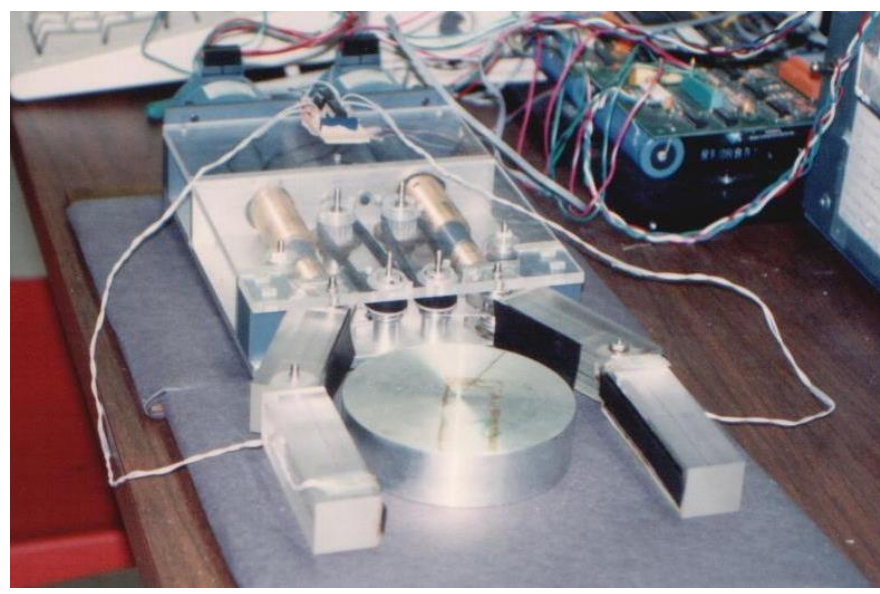

Fig. 7. Prototype gripper and a test specimen. (Note: the support surface shown in this photo was replaced by a Perspex sheet during the experimental work).

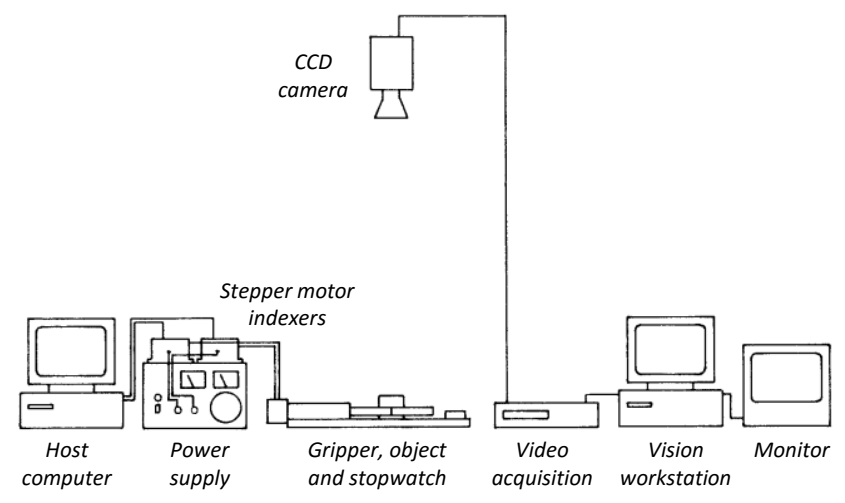

Fig. 8. Experimental set-up.

\section{Parameter normalization, metric definitions, and simulation test conditions}

The simulation program was written to take in the actual numerical parameters of the system as inputs, and is able to simulate the evolution of a grasp, and in particular to predict whether the grasp will be successful, for any set of the individual input parameters listed in Table 2 . However, in order 
to increase the scope and generality of the results of this study, it was important to investigate the parameters of the grasp in normalized form, and to test whether the results obtained were indeed independent of the absolute dimensions of the hand and object. The purpose of this section is to describe the manner in which the various parameters have been normalized, as well as to specify the general conditions applied in the simulation tests, and to define the metrics to be used to evaluate grasping performance.

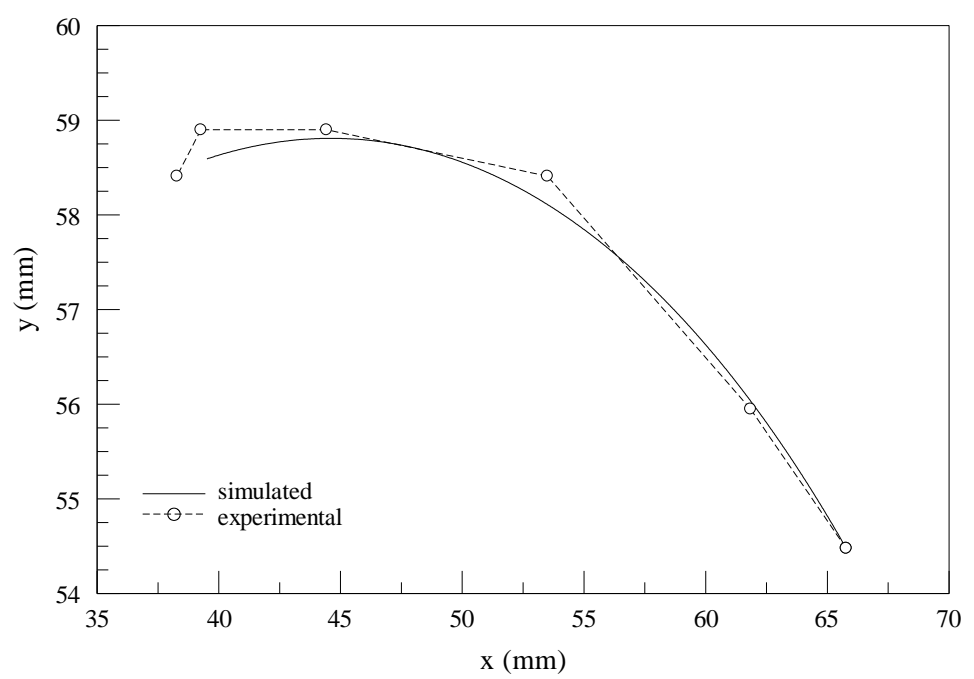

(a) Trajectory of object centroid.

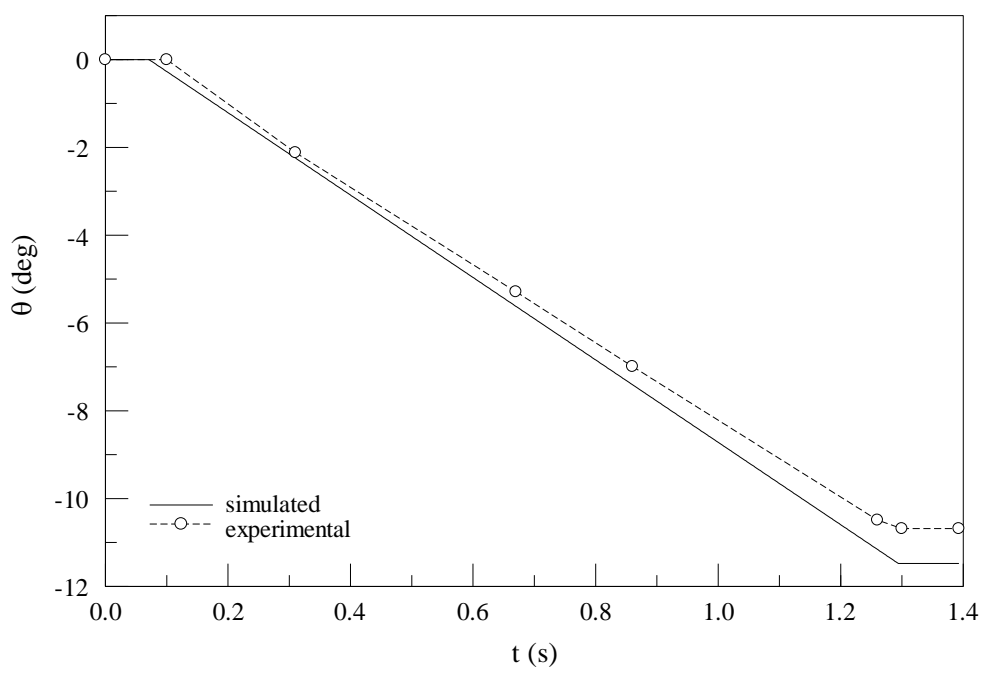

(b) Angular position of object.

Fig. 9. Comparison between experiment and simulation.

In all the simulation-based analysis that follows except where otherwise stated, all the linear dimensions have been normalized with respect to the span $S$ of the hand, defined in terms of the variables shown in Fig. 3 as follows:

$$
S \equiv 2\left(L_{L}+L_{U}\right)+P
$$

The link length ratio $L_{r}$ is defined by 


$$
L_{r} \equiv \frac{L_{L}}{L_{L}+L_{U}}
$$

For the first part of the analysis (sections 6.2 and 6.3.1) the palm width was set to $0.2 S$, resulting in a palm width to finger length ratio of 0.5 in general conformance to the findings of [1]. In section 6.3.2 the analysis was extended to consider variation in the palm width, and thus the ratio of palm width to finger length was changed accordingly.

It can be seen from Eq. (5) that the contact force $F_{N A}$ (and therefore also the joint torque) is proportional to the object mass $m$ when the other parameters are kept constant. The switch-over torque threshold at each of the lower joints and the torque limits at the upper joints were therefore normalized with respect to $m$ and set to the following values during simulation:

$$
T_{A}=T_{B}=T_{C}=T_{D}=F_{r} m g S
$$

where $F_{r}$ is a new user input parameter that specifies the ratio of the torque limits to $m g S$, and override the specific user inputs for $T_{A}, T_{B}, T_{C}$, and $T_{D}$ in the simulation. It was confirmed that in this manner the simulation results became independent of the mass $m$ of the object, as long as $F_{r}$ was high enough. During the simulation $F_{r}$ was set such that the links were able to push the object along the supporting surface during the one-contact phase of the grasp under all conditions.

\begin{tabular}{|c|c|c|c|c|c|}
\hline \multicolumn{3}{|c|}{ Hand design parameters } & \multicolumn{3}{|c|}{ Other variables of the grasp or of the simulation } \\
\hline Description & Sym. & Ref. value* & Description & Sym. & Ref. value* \\
\hline \multicolumn{3}{|l|}{ Generally fixed for a given hand: } & \multicolumn{3}{|l|}{ Object parameters: } \\
\hline Palm width & $P$ & $80 \mathrm{~mm}$ & Object radius & $R$ & $40.0 \mathrm{~mm}$ \\
\hline Lower link lengths & $L_{L}$ & $120 \mathrm{~mm}$ & Object height & $H$ & $25.4 \mathrm{~mm}$ \\
\hline Upper link lengths & $L_{U}$ & $40 \mathrm{~mm}$ & Object material density & $\rho$ & $2700 \mathrm{~kg} / \mathrm{m}^{3}$ \\
\hline Finger 1 half thickness & $E_{1}$ & $8 \mathrm{~mm}$ & Object initial $x$-position & $x_{i}$ & - \\
\hline Finger 2 half thickness & $E_{2}$ & $8 \mathrm{~mm}$ & Object initial y-position & $y_{i}$ & - \\
\hline Palm offset & $P_{O}$ & $8 \mathrm{~mm}$ & & & \\
\hline Lower links drive gear ratios & $G_{L}$ & overridden & \multicolumn{3}{|l|}{ Friction parameters: } \\
\hline \multirow[t]{2}{*}{ Upper links drive gear ratios } & $G_{U}$ & overridden & Object-link friction coefficient & $\mu_{L}$ & 0.45 \\
\hline & & & Object-surface friction coefficient & $\mu_{S}$ & 0.31 \\
\hline \multicolumn{3}{|l|}{ May be variable for a given hand: } & & & \\
\hline Link $A$ initial angle & $\alpha_{A i}$ & $0^{\circ}$ & \multicolumn{3}{|l|}{ Simulation parameter: } \\
\hline Link B initial angle & $\alpha_{B i}$ & $180^{\circ}$ & Integration time increment & $\delta \mathrm{t}$ & $0.001 \mathrm{~s}$ \\
\hline Link C initial angle & $\alpha_{C i}$ & $0^{\circ}$ & & & \\
\hline Link D initial angle & $\alpha_{D i}$ & $0^{\circ}$ & \multicolumn{3}{|c|}{ Simulation series parameters for evaluation of SGR: } \\
\hline Finger 1 motor angular velocity & $\Omega_{1}$ & overridden & Increment in $x_{i}$ & $\Delta x$ & $5 \mathrm{~mm}$ \\
\hline Finger 2 motor angular velocity & $\Omega_{2}$ & overridden & Increment in $y_{i}$ & $\Delta y$ & $5 \mathrm{~mm}$ \\
\hline Finger 1 drive switch-over threshold & $T_{A}$ & Set to $F_{r} m g P$ & & & \\
\hline Finger 2 drive switch-over threshold & $T_{B}$ & Set to $F_{r} m g P$ & \multicolumn{3}{|l|}{ New overriding parameters: } \\
\hline Finger 1 upper link torque limit & $T_{C}$ & Set to $F_{r} m g P$ & Joint speeds & $\omega$ & $1 \mathrm{rad} / \mathrm{s}$ \\
\hline Finger 2 upper link torque limit & $T_{D}$ & Set to $F_{r} m g P$ & Joint torque limit factor & $F_{r}$ & 0.4 \\
\hline
\end{tabular}

Table 2 - User input data for simulation.

*The indicated units refer to those input by the user. The simulation works exclusively in SI units. 
The angular speeds of the moving joints were assigned a common value of $\omega$ (in the appropriate directions), which is a new user input parameter that overrides the specific user inputs for $\Omega_{1}, \Omega_{2}, G_{L}$, and $G_{U}$ listed in Table 2. In all the simulations, the link offsets $E_{1}$ and $E_{2}$ and the palm offset $P_{O}$ were assigned equal values, here referred to as the offset parameter $E$. In order to conform to the approach taken in other studies (e.g. [8]), the finger interference checks (which are a feature of the present simulation program) were disabled, and it was assumed that the links would not interfere with each other. In a real gripper this can be achieved by designing the fingers to have a slight offset from each other outside the plane.

In the present work the successful grasp range (SGR) for a specific set of parameters for hand, object and friction is defined in normalized form as the ratio

$$
\mathrm{SGR} \equiv \frac{A_{\mathrm{SG}}}{A_{\mathrm{WS}}}=\frac{1}{A_{\mathrm{WS}}} \iint_{A_{\mathrm{SG}}} d y d x
$$

where $A_{\mathrm{SG}}$ is the area in the workspace of the hand, within which the object (centre) can be initially located (relative to the fixed palm, and without interfering with the links or the palm), and within which a successful enveloping grasp of the object is achieved; and where $A_{\text {WS }}$ is the workspace area of the hand. In order to ensure a fair comparison between the various parameter sets, the initial link angles for all the simulations in this study were set as shown in Fig. 10, such that the hand was fully open at full span, with both fingers positioned collinear with the palm. Furthermore, in order to be able to compare directly the SGR for different hand designs (even where the ratio of $P$ to $S$ is changed), the workspace of the hand is defined by the shaded region in the figure, and has an area of $0.125 \pi S^{2}$. This represents the largest workspace theoretically achievable for a hand of span $S$, obtained when both $P$ and $E$ are equal to zero. The SGR is evaluated by running a series of simulations at various increments of $x_{i}$ and $y_{i}$. Specifically, the successful grasp range is evaluated as

$$
\mathrm{SGR}=\frac{1}{0.125 \pi S^{2}} \sum_{x_{i}, y_{i}} I \Delta y \Delta x
$$

where $\Delta x$ and $\Delta y$ are the increments in $x_{i}$ and $y_{i}$ at which the simulations are carried out, and $I$ has a value of 1 if an enveloping grasp is achieved (for the considered values of $x_{i}$ and $y_{i}$ ), and of 0 otherwise. In the simulations satisfactory convergence was achieved when $\Delta x$ and $\Delta y$ were each set to $0.0125 S$.

In the optimization exercises, the grasping performance is evaluated through the SGR (as computed by Eq. (21) above), and/or the grasping ability (see [10] and section 1 above) defined as

$$
Q_{\text {grasp }}=\frac{\pi \Delta R}{S}
$$

An important outcome of the above described test conditions is that in all of the successful grasps, the final position of the object will be on the hand centerline as required by the research objectives. The two fingers of the hand will move in a symmetric manner throughout the grasping process, subject to the assumptions stated in section 2.1. This outcome contrasts with earlier results found in the literature (e.g. $[1,8,13])$ where the immovable nature of the object in the static analyses forces the 
gripper links to assume a non-symmetric configuration for situations where the initial position of the object is non-symmetric (such analyses may be applicable to, say, grasping the handle of a heavy box). In the present work, the object is allowed to move during the grasping process, and thus the final grasp, if successful, is symmetric if the following three conditions are true: (i) the starting configuration of the gripper is symmetric; (ii) joint angular speeds are equal; and (iii) the joints are sufficiently powered to be able to push the object along the supporting surface during the one-contact phase of the grasp under all conditions. This analysis would be applicable to real world scenarios such as those suggested in section 1 . It is also noted that since the simulation exercises reported in this work all involve simultaneous and symmetrical finger movements, as well as simultaneous switching over of the drives, the entire optimization study of the present work is applicable also to hands of the type being analyzed that are driven symmetrically by a single actuator instead of by two actuators.

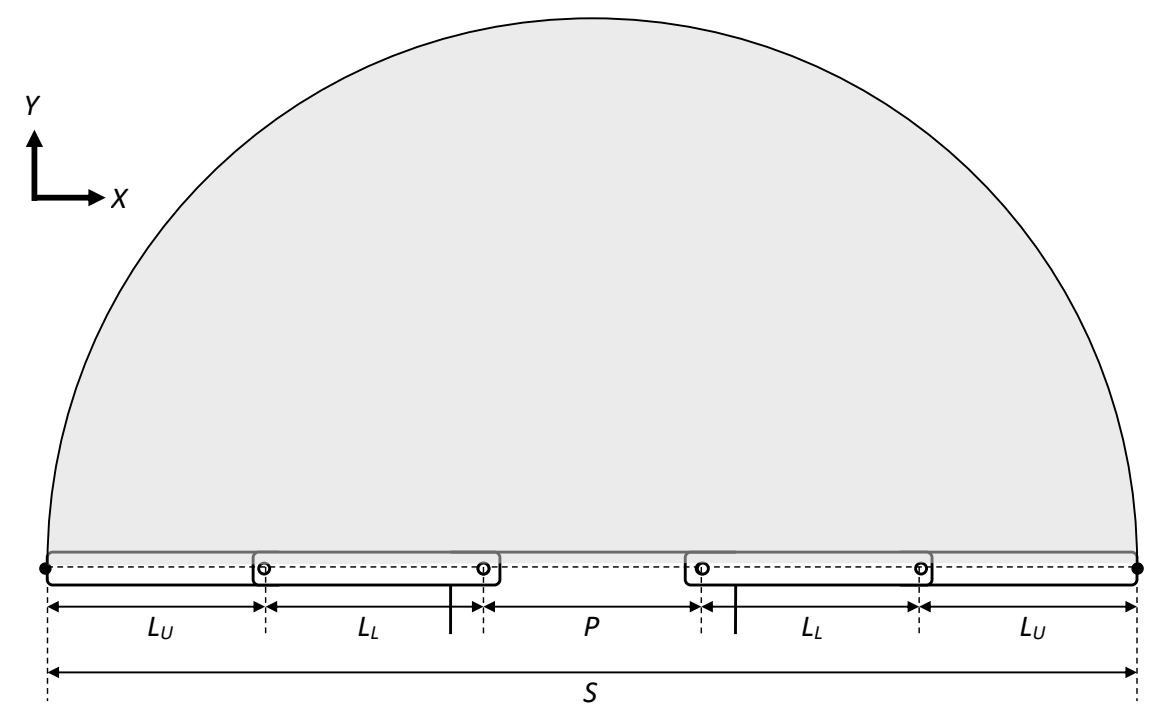

Fig. 10. Workspace of the robot gripper.

\section{Parameter space study and design optimization}

\subsection{Overview}

It is recalled that the objectives of this section were (i) to use the simulation to understand the effects of various design and application parameters of the hand on grasping performance; and (ii) to exploit this understanding by suggesting ways in which the design of the system can be improved. The system parameters under study are the link length ratio $L_{r}$ (see Eq. (18)), the offset parameter $E$ (see section $5)$, the friction coefficient between the object and the supporting surface $\mu_{S}$, and the link angular speed $\omega$.

In the first part of this study (section 6.2) the object radius is set to $R=0.5 P$, hereinafter referred to as the characteristic radius $R_{\text {char }}$ of the object for a hand of palm width $P$. The objective of this part of the study is to investigate the effect of $L_{r}, E, \mu_{S}$ and $\omega$ on SGR, and leads to a set of system design guidelines for the case most widely considered in the literature (i.e. finger length $\approx 2 P$ and $R \approx R_{\text {char }}$; see Fig. 1). It is noted that, as indicated in subsections 6.2 .1 to 6.2 .4 , the parameters under study were varied simultaneously where there was the possibility that these could be coupled, in order to ensure that the result represented a true optimum. 
In the second part of the study (section 6.3), the effects of varying $R$ and $P$ are investigated, leading to a suggestion for further improvement in the design of the hand.

\subsection{Parametric study and optimization at the object characteristic radius}

\subsubsection{Link length ratio}

\section{Method and Results}

With the exception of $L_{L}$ and $L_{U}$, the simulation parameters were set to their reference values as given in Table 2. The SGR was evaluated for various values of $L_{r}$ between 0.05 and 1.00 , and the results are given in Fig. 11 . The highest SGR was obtained at approximately $L_{r}=0.75$, indicating that the optimum ratio between the lower and upper link lengths under the specified conditions is $3: 1$. The reference values (see Table 2) for $L_{L}$ and $L_{U}$ for subsequent investigations were set in accordance with this result. Fig. 11 also shows the average time for a successful enveloping grasp as a function of $L_{r}$, with the slowest time occurring at approximately $L_{r}=0.65$.

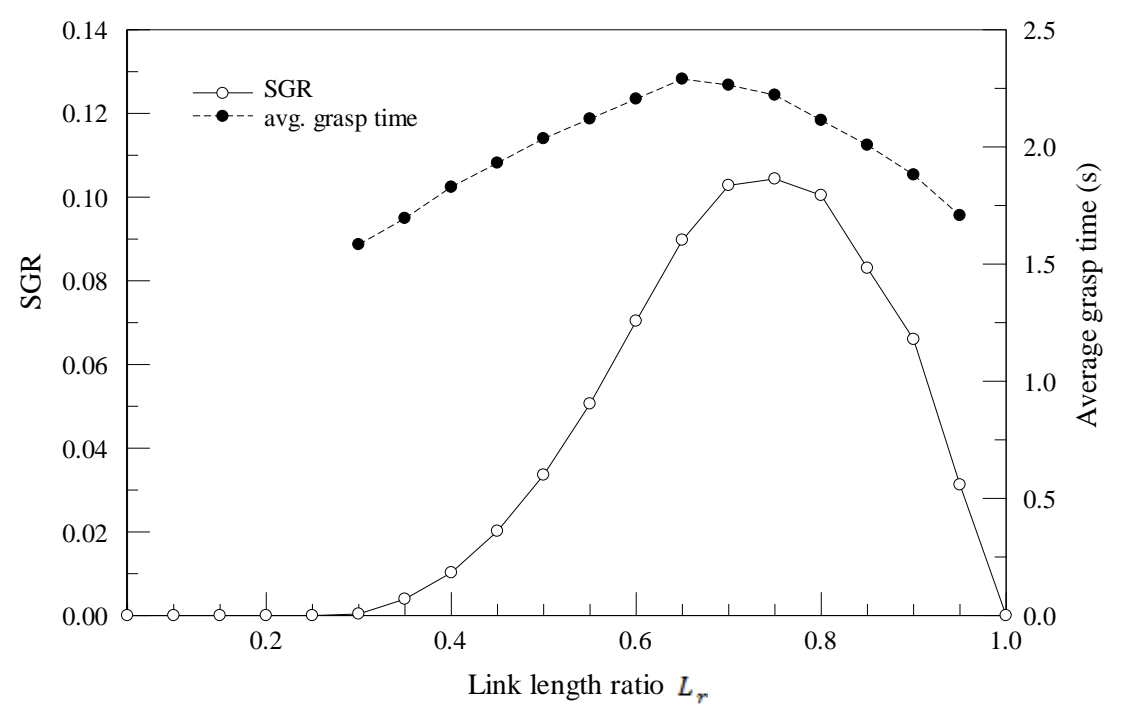

Fig. 11. Effect of link length ratio on grasping performance.

\section{Explanation}

It is recalled that due to the symmetries imposed in this work, a successful enveloping grasp with $R=$ $R_{\text {char }}$ will always satisfy the conditions that (i) the object centroid will lie on the centre-line of the hand between the two fingers, and (ii) each of the four links will touch the object with the two fingers assuming symmetrical postures. While at first intuition this might suggest that all four links should be of equal length to maximize SGR, this is not the case. For cases where the initial position of the object centroid is not on the hand centre-line (i.e. for the vast majority of cases in the evaluation of SGR) the object will roll up the finger after first contact as it is dragged towards the centre-line by the contacting finger. In order to eventually be able to satisfy condition (ii) above, it is important that the object does not roll onto the upper link before contact is made with the second finger. This effect biases the results towards an optimum $L_{r}$ value that is higher than 0.5 for maximum SGR.

\subsubsection{Hand offset parameter}




\section{Method and Results}

With the exception of $E_{1}, E_{2}, P_{o}, L_{L}$ and $L_{U}$, the simulation parameters were set to their reference values as given in Table 2. The SGR was evaluated for various values of $E$ between 0 and $0.06 S$, in each case for values of $L_{r}$ between 0.05 and 1.00. A plot of peak SGR versus $E$ is given in Fig. 12. For each value of $E$, the peak SGR was found to occur at approximately $L_{r}=0.75$. The results in Fig. 12 indicate that the hand should be designed such that $E$ is as small as possible in order to maximize the successful grasp range. In practice, there is a physical lower limit on $E$, and as such in this study the reference value for this parameter has been set to $0.02 S$. For the parameter reference values given in Table 2, it was found that

$$
\mathrm{SGR}=0.105
$$

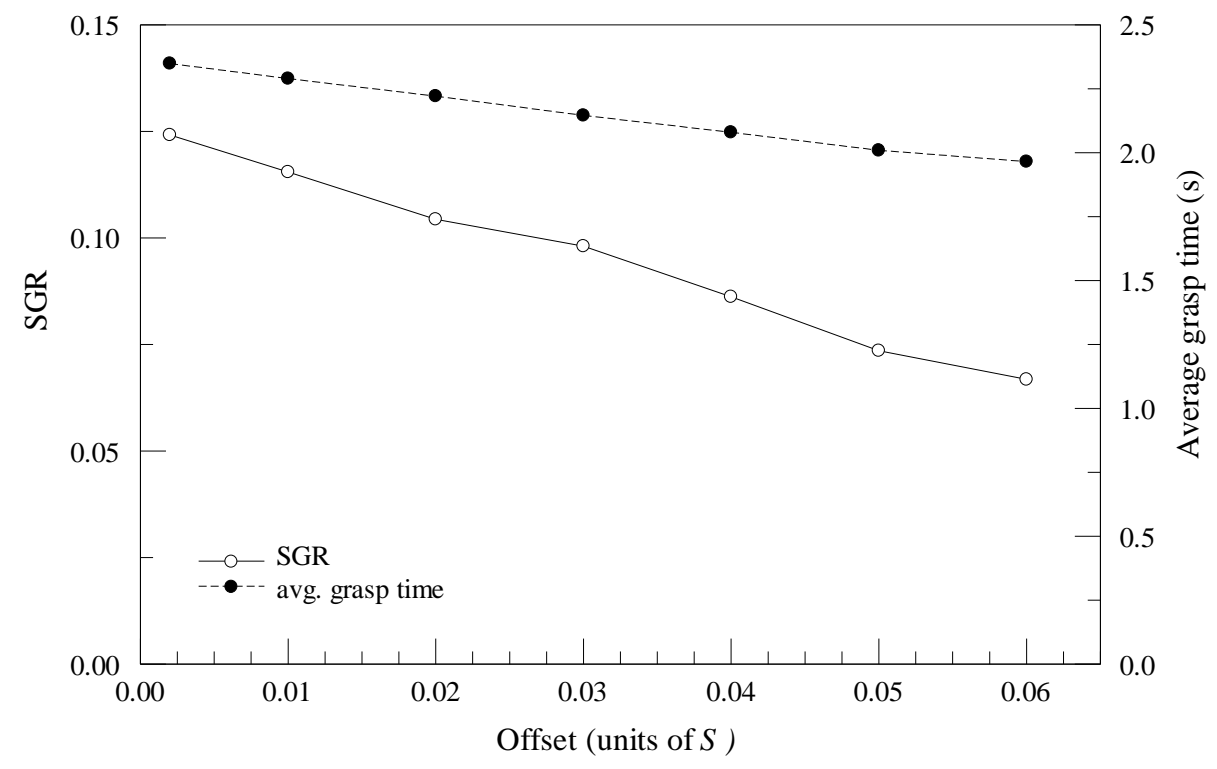

Fig. 12. Effect of the offset parameter on grasping performance.

\section{Explanation}

The main reason for this result is a geometrical effect as shown by Eq. (9) and explained at the end of section 2.2.2. A higher $E$ results in a higher $\sin \varepsilon$ and therefore in a higher object rolling velocity during one-link contact. This is detrimental to SGR for the reasons explained in section 6.2.1 above. A higher $E$ also reduces the effective workspace of the hand resulting in a smaller area where the object can be placed prior to the grasp without interfering with the links. 


\subsubsection{Friction coefficient between the object and the supporting surface}

\section{Method and Results}

With the exception of $\mu_{S}, L_{L}$ and $L_{U}$, the simulation parameters were set to their reference values as given in Table 2. The SGR was evaluated for various values of $\mu_{S}$ between 0 and 1 , in each case for values of $L_{r}$ between 0.05 and 1.00. Plots of SGR and of grasping time against $\mu_{S}$ are shown in Fig. 13. It is noted that for the two data points with $\mu_{S}>0.8$ in the figure, the joint torque limit factor $F_{r}$ was increased to 0.8 in order to ensure that the links were able to push the object along the supporting surface during the one-contact phase of the grasp. The data indicate that as long as the link torque limits are sufficiently high as described, increasing the friction with the supporting surface has the effect of improving the SGR and also of slightly reducing the average time taken to grasp the object. For all the data points in Fig. 13, the peak SGR was found to occur at $L_{r} \simeq 0.75$.

\section{Explanation}

A larger $\mu_{S}$ results in higher $T_{f}$ and $F_{f}$, and reduces angular acceleration of the object up the link. This reduces the number of cases where the object rolls up to the outer link before contact is made with the second finger, and therefore results in an increase in SGR. As will be seen in section 6.2.4, this situation is true only for the case where $\omega$ is greater than a critical value.

\subsubsection{Link angular speed}

\section{Method and Results}

With the exception of $\omega, \mu_{S}, L_{L}$ and $L_{U}$, the simulation parameters were set to their reference values as given in Table 2. The SGR was evaluated for various values of $\omega$ and $\mu_{S}$, in each case for values of $L_{r}$ between 0.05 and 1.00. Plots of SGR and of grasping time against $\omega$ are shown in Fig. 14. It is seen that for low values of $\omega$ the SGR is not dependent on the link velocity, but then starts to fall off beyond a critical value of $\omega$. It is also seen that the highest angular speed that sustains maximum SGR increases as $\mu_{S}$ is increased. For all the data points in Fig. 14, the peak SGR was found to occur at $L_{r} \simeq 0.75$.

\section{Explanation}

During the one-contact phase of the grasps for low $\omega$ the centrifugal force of the object is not enough to overcome the frictional force and torque against the supporting surface, and hence there is no angular acceleration of the object. The initial object velocity is proportional to the link velocity as per Eq. (9), and as long as there is no slip at the link, the object movement relative to the link is determined by geometrical constraints that are non-dynamic and independent of link velocity. As $\omega$ increases beyond the critical value, acceleration of the object during one-link contact with a lower link causes some of the grasps to become unsuccessful as the object rides up to the upper link before contact is made with the second finger, therefore making it impossible to obtain an enveloping grasp. This results in a deficit in the SGR. As $\mu_{S}$ increases, higher values of $T_{f}$ and $F_{f}$ push the critical value of $\omega$ upwards. For the reference conditions listed in Table 2 the critical value of $\omega$ is about $0.6 \mathrm{rad} / \mathrm{s}$. 


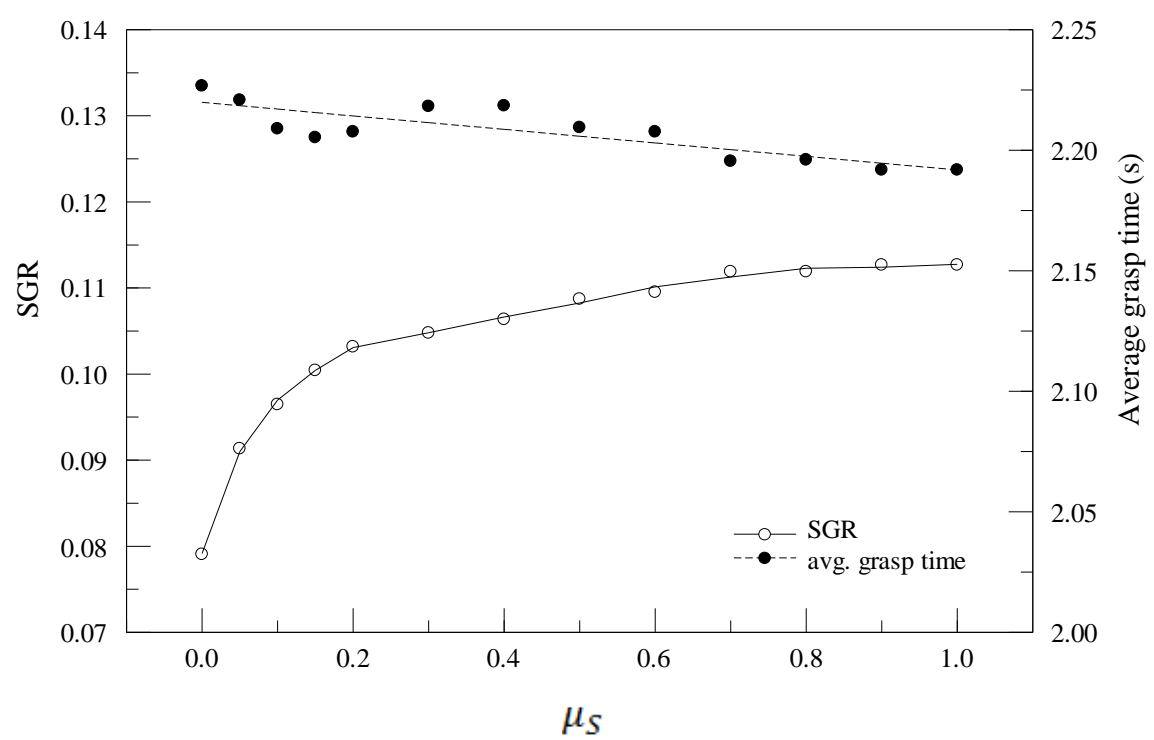

Fig. 13. Effect of the surface friction on grasping performance.

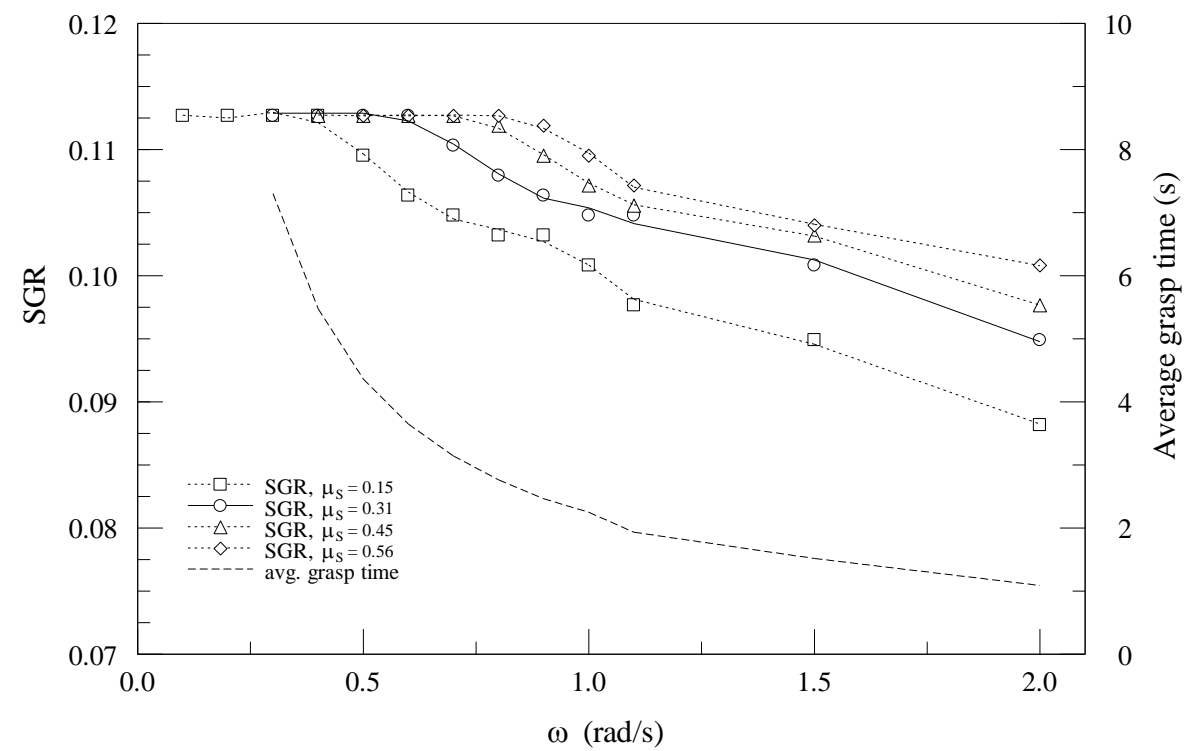

Fig. 14. Effect of link speed on grasping performance.

\subsubsection{System design guidelines}

The investigations of sections 6.2.1 to 6.2.4 lead to several design guidelines for optimizing the successful grasp range for a basic planar underactuated hand of the type shown in Fig. 1 and described in sections 1 and 2.1, where the finger length is twice the palm width, in grasping an object of characteristic radius. Specifically: (i) The ratio of lower to upper link length should be 3:1; (ii) the offset dimensions of the fingers and palm should be as small as possible; (iii) the friction coefficient between the object and supporting surface should be maximized provided that smooth object sliding on the surface is feasible and the joint torque limits of the hand permit pushing of the object under one-link 
contact in all situations; and (iv) the link speeds should be set at the maximum value that sustains the maximum SGR under these conditions, as long as the average grasping time is acceptable.

In a real world scenario based on the reference aluminum objects described in section 4 , where the objective may be to maximize the tolerance in initial object positioning for successful grasping, the robot hand should be redesigned to satisfy conditions (i) and (ii) above. During the experimental phase of the present study, a number of tests were conducted to measure the friction coefficient between the aluminum objects used in the experimentation and various support surface materials (aluminium, nylon and Perspex). The highest average reading among the tested materials was obtained for aluminum on nylon with $\mu_{S}=0.56$. Thus in the considered real world scenario, the supporting surface would be constructed out of nylon to satisfy condition (iii) and to optimize the system performance. The motor speeds of the hand would then be set to give joint speeds of $0.8 \mathrm{rad} / \mathrm{s}$ in accordance with Fig. 14, thereby satisfying condition (iv).

The SGR for the scenario described above was evaluated using the simulation software and was found to equal 0.112 , as illustrated in Fig. 15 . The shaded region in the figure illustrates the starting positions of the object centre that would result in successful enveloping grasps. The crescent shape of this region is characteristic of these tests and is explained by the fact that for starting positions away from the hand centre-line, candidates for a successful grasp must be placed at lower $y$ in order to arrive within the appropriate $y$-range on the hand centre-line, after rolling up the link during one-link contact. The low value of SGR is due to two reasons: (i) a large percentage of candidate object starting positions within the workspace are eliminated due to interference between the object and the hand structure; and (ii) as per the definition of SGR successful grasps must envelope the object, and thus the equilibrium grasp outcomes that are non-enveloping are eliminated.

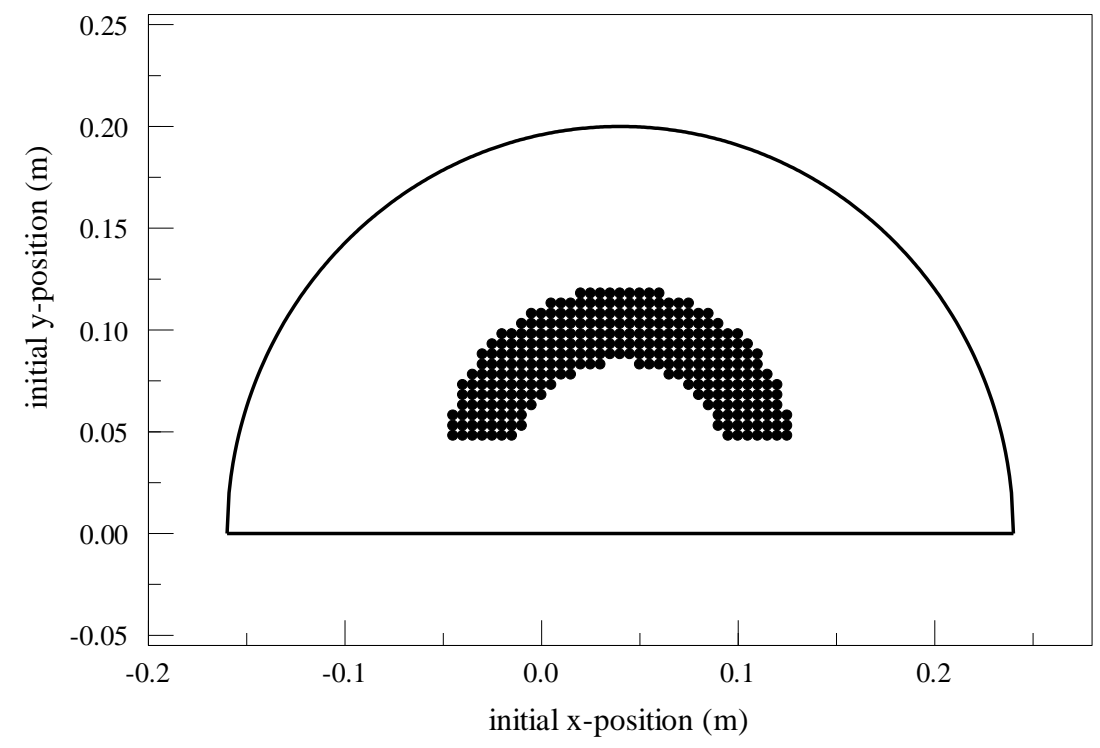

Fig. 15. An SGR region equalling $11.2 \%$ of the hand workspace. 


\subsection{Variability of object size}

\subsubsection{Grasping ability of the hand}

\section{Method and results}

The simulation parameters were set to their reference values as given in Table 2, and the simulation was run for different values of object radius $R$. It was noted that for all the values of $R$, the SGR peaked at approximately $L_{r}=0.75$, indicating that this optimum ratio for link lengths is valid even for $R \neq$ $R_{\text {char }}$. The key results of these runs are presented in Fig. 16, where the SGR is plotted against radius, normalized with respect to $1 / 2[P-2 E]$. It is seen that the $S G R$ is a maximum for an object radius that is just above a critical radius $R_{\text {crit }}$, given by

$$
R_{\text {crit }}=\frac{P-2 E}{2}
$$

\section{Explanation}

As $R$ increases beyond $R_{\text {crit }}$, the SGR decreases gradually (due mainly to a lower percentage of the workspace being available for non-interfering initial positions of the object), whereas for objects of radius less than $R_{\text {crit }}$ the hand is not able to achieve an enveloping grasp. The significance of this critical radius is illustrated in Fig. 17 . At or below $R_{\text {crit }}$ it is not possible for all pairs of adjacent links in a three or four contact grasp to subtend an angle of less than $\pi$. This type of hand is therefore not suitable for grasping of objects smaller than the critical radius.

For the hand under consideration, with $P=0.2 S, E=0.02 S$, and therefore $R_{\text {crit }}=0.08 S$, the SGR is non-zero if $0.08 S<R<\sim 0.26 S$. The grasping ability of the hand (Eq. (22)) is therefore approximately equal to $0.18 \pi$.

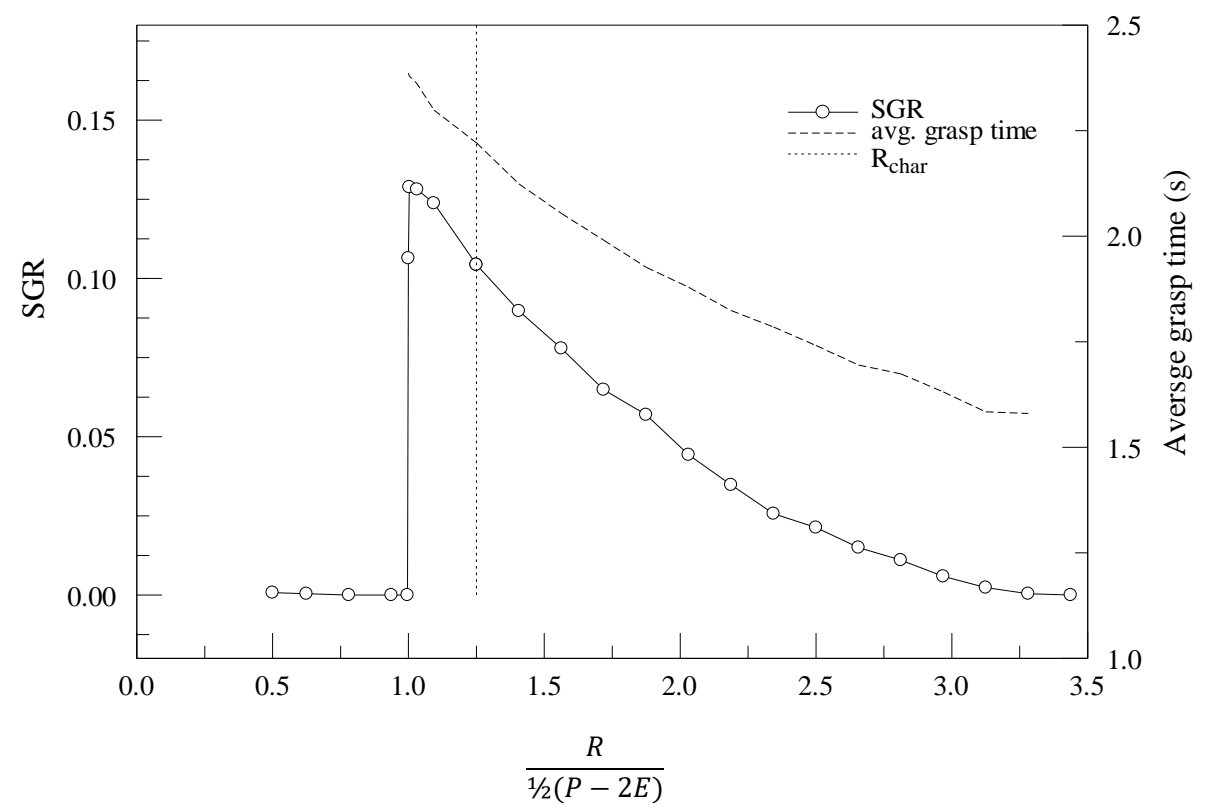

Fig. 16. Grasping performance for different object radii. 


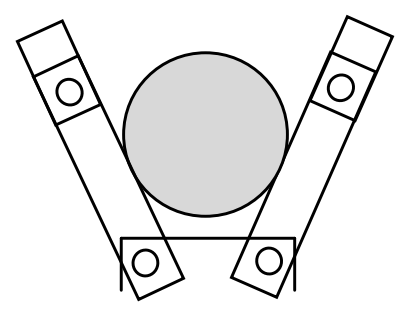

(a)

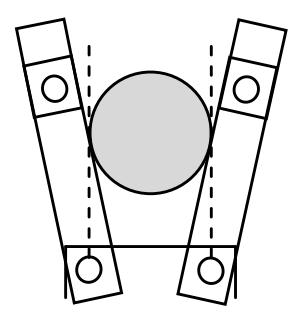

(b)

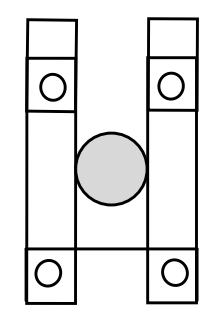

(c)

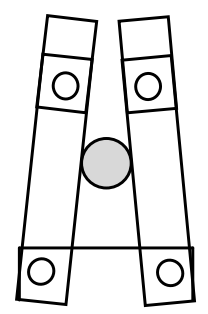

(d)

Fig. 17. Object radii relative to palm width: (a) $R>R_{\text {char }}$, (b) $R=R_{\text {char }}$, (c) $R=R_{\text {crit }}$, (d) $R<R_{\text {crit }}$.

\subsubsection{Optimizing the SGR and the grasping ability for a given span of hand}

The results in Fig. 16 suggest that for a given span of hand, the grasping ability may be improved if the palm width $P$ is reduced, giving smaller $R_{\text {crit }}$ and potentially a wider range of graspable object sizes. This hypothesis was tested using the simulation software. The simulation was run for various values of $P, R$ and $L_{r}$, with the other input variables set as indicated in Table 2. It was found that in all cases the peak SGR occurred at around $L_{r}=0.75$ as for the previously analyzed hand. The results at this value of $L_{r}$ are given in Fig. 18, and the variation of $Q_{\text {grasp }}$ with $P$ is shown in Fig. 19. It is seen that for a given span of hand, a decrease in $P$ is associated with increases in both SGR and $Q_{\text {grasp }}$.

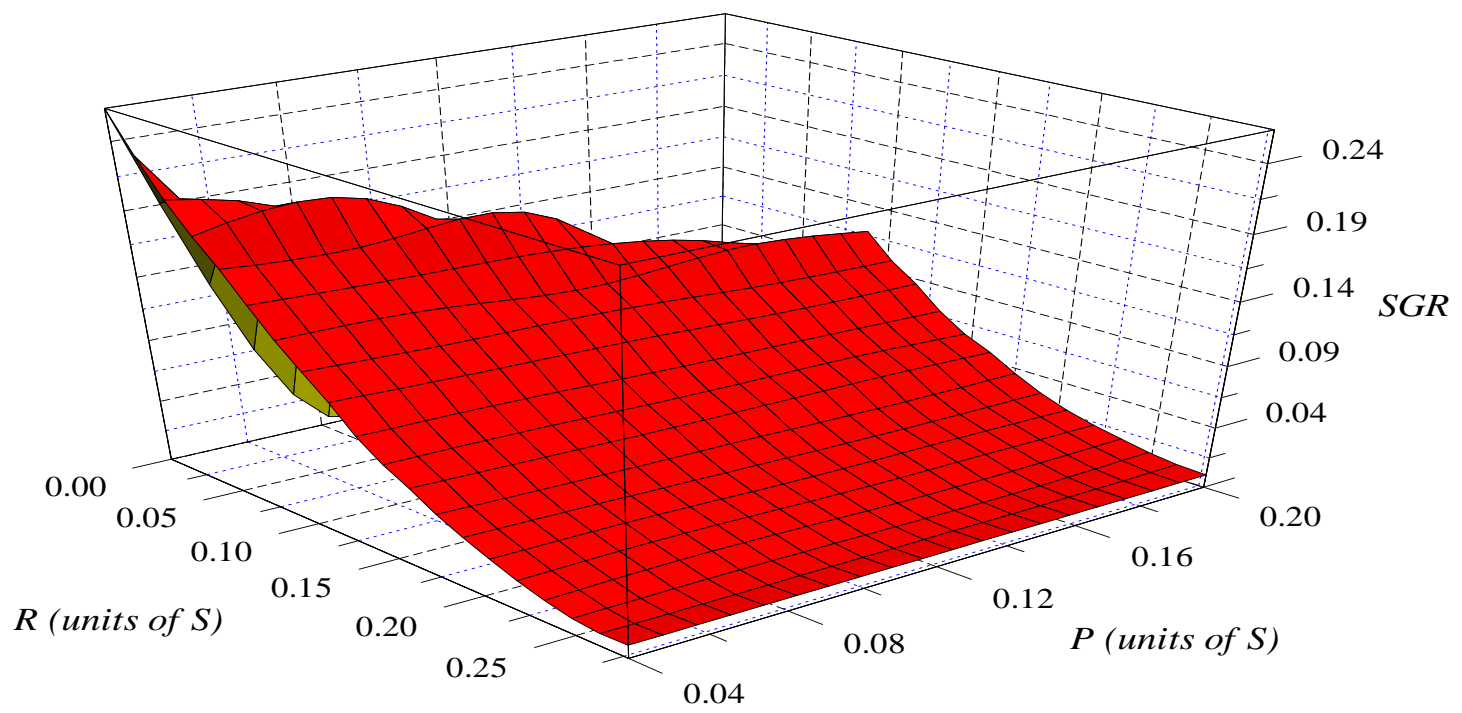

Fig. 18. Variation of peak SGR with $P$ and $R$ at $L_{r}=0.75$ 


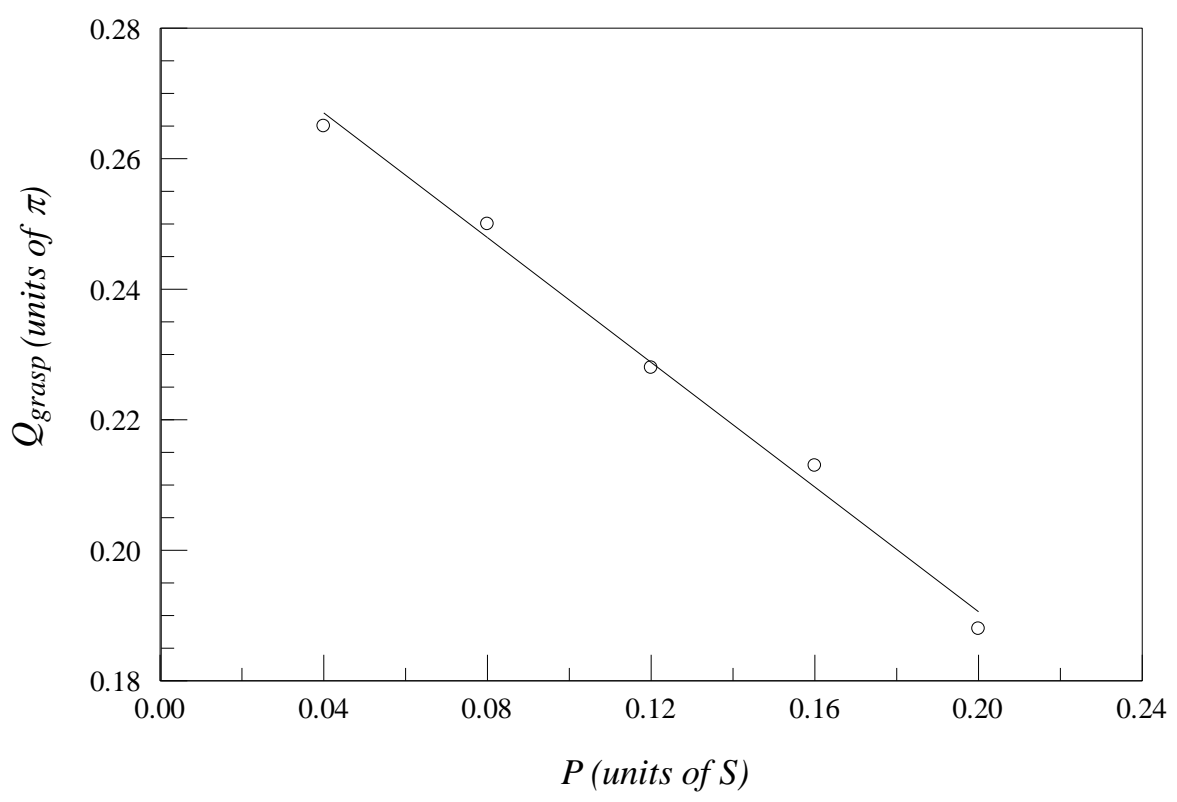

Fig. 19. Variation of $Q_{\text {grasp }}$ with $P$

The main significance of these results is the indication that in order to optimize the successful grasp range and the grasping ability for a given span of underactuated hand of the conceptual type illustrated in Fig. 1, the ratio between the palm and finger lengths should be set to as low a value as possible, rather than to the common value of around 0.5 reported in [1] and normally analyzed in the literature (including in sections 6.2 and 6.3.1 of the present work). An improved hand design that is based on the results of this subsection, is suggested in Fig. 20, with $P=2 E$ and $L_{r}=0.75$, which significantly increases $Q_{\text {grasp }}$, and to a lesser extent also SGR.

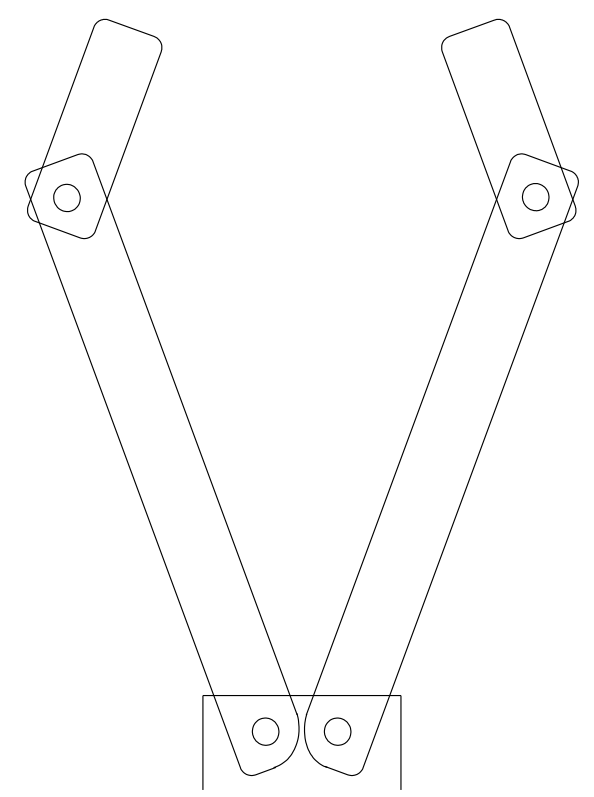

Fig. 20. An improved hand design that maximizes SGR and $Q_{\text {grasp }}$. 
This paper is proposing two significant and visually very distinct changes to the geometry of planar, underactuated, two-finger, four-joint hands that are normally presented and analyzed in the literature, as can be seen by comparing Fig. 1 and Fig 20. The first of these changes is the significantly shorter length of the upper links relative to the lower links. The second change involves a much reduced palm to finger length ratio. Other recommended design guidelines are that the grasping faces of the links should be offset by as small a distance as possible from the radial sweep of the links (i.e. $E$ in Fig. 3 should be as small as possible); that the friction coefficient between the object and the horizontal supporting surface should be increased (to the maximum value that allows the gripper joints to have sufficient power to push the object along the surface during grasping in all situations); and that the joint speed has an optimum value that is a function of this friction coefficient.

These results may be somewhat unexpected at first consideration, however it is shown in section 6 that every result obtained is clearly understood and can be explained in retrospect. It is emphasized that the grasping problem analyzed here differs fundamentally from most previous studies in that the object is allowed to move within the workspace as it is being grasped, and in particular is always manipulated to the hand centerline. Furthermore, unlike previous studies this work has dealt exclusively with a triggered-type underactuated hand that has nonbackdrivable and overpowered joints. An investigation of the extent to which each of these differences contributed to the nature of the results is beyond the scope of the present work.

It is worth rationalizing and demonstrating some of the results obtained in practical terms, by comparing the performance of two grippers of equal span $S$, one having the geometry of Fig. 1 (here called the conventional design), and the other having the geometry of Fig. 20 (the new design). Fig. 21 shows the SGR, and typical final configurations, of the two grippers in grasping objects of sizes $R=$ $0.15 S, R=0.10 S$ (which is the characteristic radius for the conventional gripper), and $R=0.05 S$, respectively. It can be seen in Figs 21(a) and (d) that for the larger object sizes, the SGR for the new design is significantly larger than that for the conventional design, and that in both sets of cases the object can be securely grasped (Figs 21 (b), (c), (e), (f)). For the small object, the new gripper has a significantly large SGR (Fig. 21(g)) and can grasp the object securely (Fig. 21(i)), while the conventional gripper is unable to grasp this object in an enveloping way (Fig. 21(h)).

Fig. 22 compares the largest and smallest objects that can be grasped by the two designs (reflected in the performance parameter $Q_{\text {grasp }}$ ). For the conventional design, the maximum size of the object is limited by interference with the palm, and is approximately equal to $0.2 S$ (Fig. 22(a)). For the new design, the maximum object size is limited by the reach of the upper links in attaining tangential contact, and is approximately equal to $0.25 S$ (Fig. 22(b)). The minimum object sizes for the two grippers are given by their respective $R_{\text {crit }}$ values, and are equal to (or, more precisely, are just greater than) $0.08 S$ for the conventional design (Fig. 22 (c)), and zero for the new design (Fig. 22(d) shows an object of radius $0.01 S$ being grasped). In all of these cases the new design has better performance as measured by the metrics applied in this work.

Although the projected performance of the hand designs have been evaluated based on established metrics found in the literature (successful grasp range and ability to grasp), it remains important to 


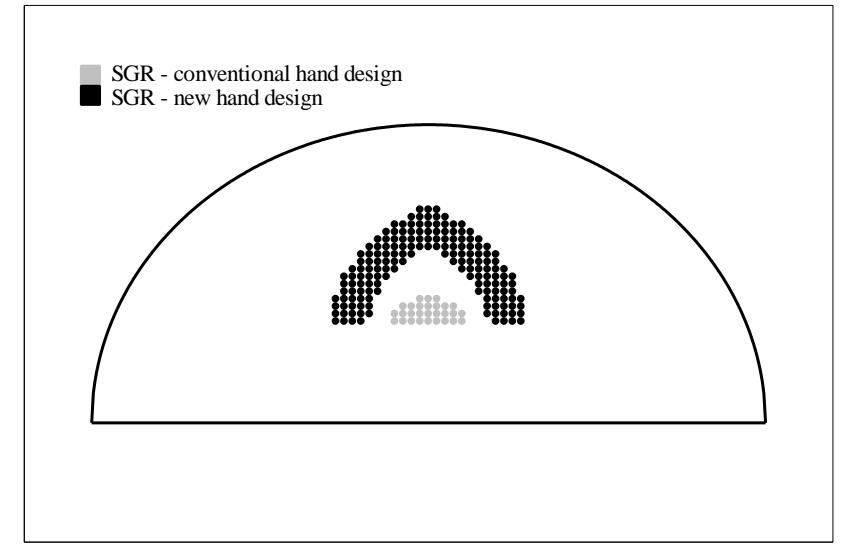

(a)

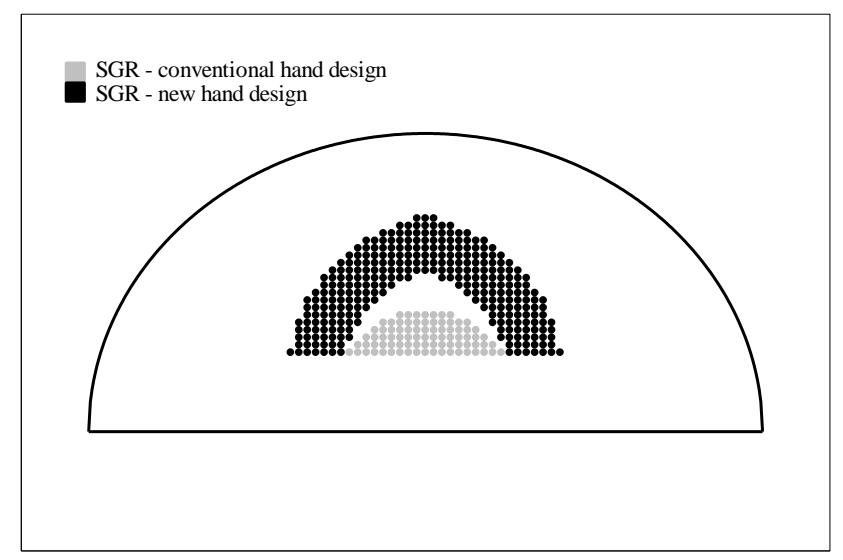

(d)

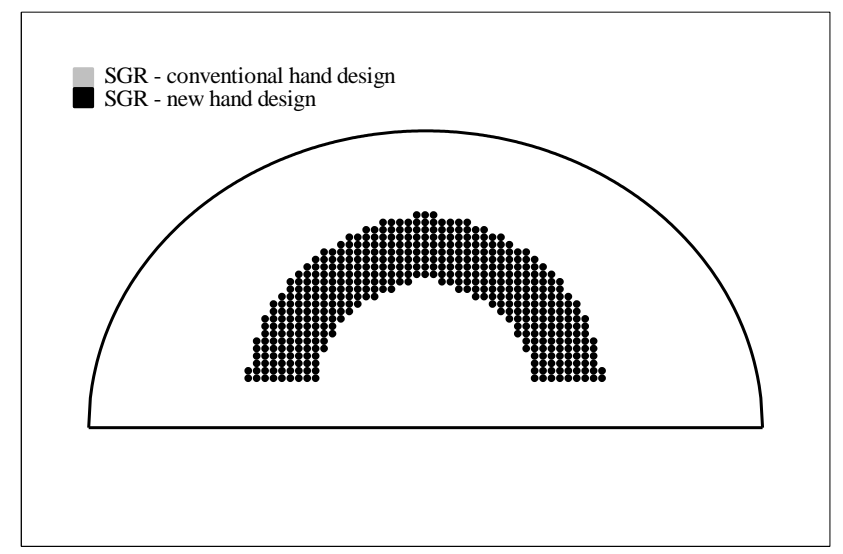

(g)

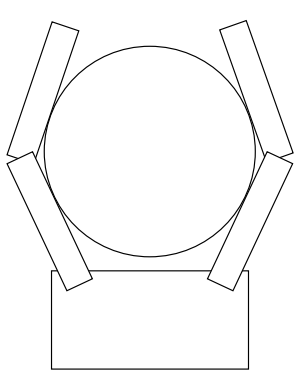

(b)

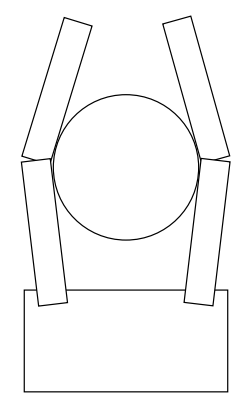

(e)

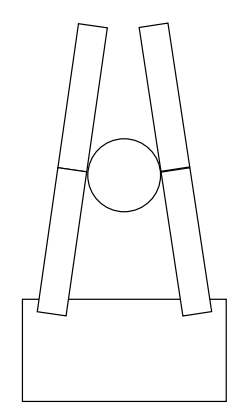

(h)

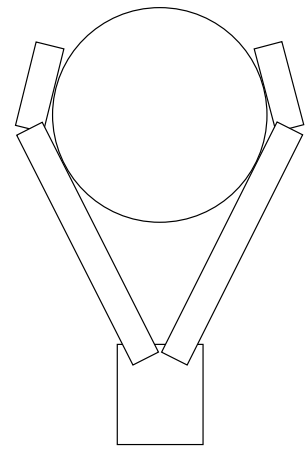

(c)

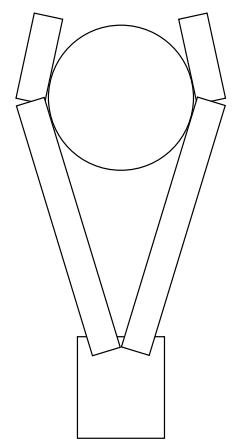

(f)

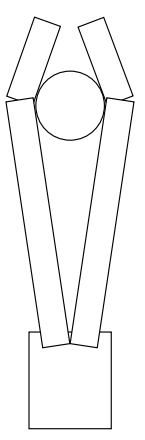

(i)

Fig. 21. SGR comparisons and typical final grasp configurations by the conventional and new gripper designs, for various object sizes.

interpret and apply the results carefully. Planar underactuated hands are often designed with the primary objective of adaptability to a wide range of object shapes (of the same approximate size) not of object sizes, and furthermore it is often intended that through the structure of the environment or through visual guidance the hand can approach the object such that it is approximately centred within the hand workspace prior to the grasping operation. In such situations many of the results of this work do not apply. In cases where there is prior knowledge of object size but not of initial position, the 


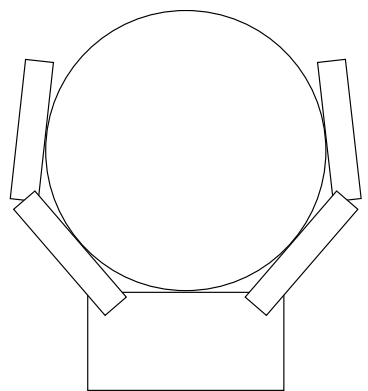

(a)

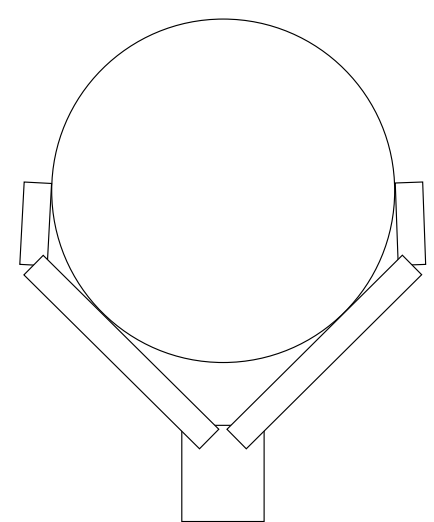

(b)

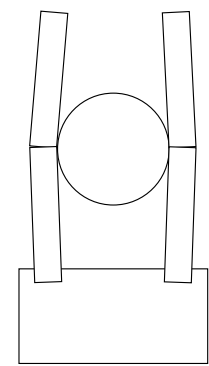

(c)

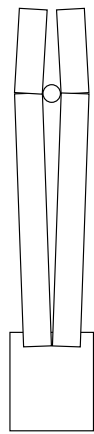

(d)

Fig. 22. Comparison between the conventional and new gripper designs with respect to $Q_{\text {grasp }}$

results of section 6.2 apply. Finally, in situations where there is no prior knowledge of either the size or the initial position of the object, the results of section 6.3 apply.

Finally, it is noted from Figs 21(a), (d), and (g) that the SGR for the new design, while relatively larger, does not have an ideal shape for the projected practical applications. This is because the positional error of an object to be grasped is likely to be isotropically distributed, and the SGR of the gripper should therefore ideally have a round shape, or as close to one as possible, for maximum benefit. The crescent shape of the SGR reduces to some extent the impact of the gains obtained in the practical situation, at least with respect to the SGR. An approach to this problem might involve accepting all force closure grasps, including the non-enveloping ones, as successful, and then comparing the now more heavily populated SGR plots of various gripper designs to determine new optimal parameters. Such an exercise would still need to take into account, with appropriate weighting, that an enveloping grasp is better than a non-enveloping force closure grasp.

\section{Conclusion}

This paper made several contributions to the analysis and optimization of underactuated robot hands. First, a new quasi-dynamic analysis of the grasping process by a planar, four-joint underactuated hand was presented. Unlike previous analyses for this type of hand, the present paper set emphasis on object movement during the grasping process, and also took into account frictional contact with a supporting surface. The present version of the analysis is applicable to triggered-type hands with nonbackdrivable joints. Second, an extensive and versatile simulation program, based on the analysis, was developed to study the effects of various hand and object parameters on the grasping process. A prototype hand was built and the simulation study was experimentally validated in part of the design space using the prototype. Third, an extensive study and optimization exercise was carried out, focusing on the basic underactuated hand that is currently and predominantly discussed in the literature. Important findings on the influence of link length ratio, link offset value, link angular speed, friction with the supporting surface, object mass and object size on the successful grasp range were presented, and the results were used to establish a set of new design guidelines for a hand of this type. Following through the logical path uncovered by these results, a new and improved design approach for hands of this type was suggested in order to improve performance as quantified by the 
successful grasp range and the grasping ability of the hand. Finally, the new design was discussed critically.

In particular, the results have indicated that in order to maximize the successful grasp range of a hand of this type for applications where there will be limited information and / or variability of the size and precise initial location of the object to be grasped, the optimal hand design would involve inner to outer phalange size ratios of approximately 3:1, and inner phalange joints that are very close to each other. Furthermore, this work has highlighted the non-negligible effects of the link thickness, of the joint speeds, and of the friction coefficient between the object and its plane horizontal supporting surface, on the evolution and outcome of the grasping process.

\section{References}

[1] Laliberté, T. and Gosselin, C. M., 1998, "Simulation and design of underactuated mechanical hands," Mechanisms and Machine Theory, 33(1/2), pp. 39-57.

[2] Saliba, M. A. and de Silva, C. W., 1991, "An Innovative Robotic Gripper for Grasping and Handling Research," Proceedings of the IEEE International Conference on Industrial Electronics, Control and Instrumentation (IECON'91), Kobe, Japan, vol. 2, pp. 975-979.

[3] Birglen, L., Laliberté, T. and Gosselin, C. M., 2008, "Underactuated Robot Hands," Springer Tracts in Advanced Robotics, 40, pp. 17-31.

[4] Bégoc, V., Krut, S., Dombre, E., Durand, C. and Pierrot, F., 2007, "Mechanical design of a new pneumatically driven underactuated hand," Proceedings of the IEEE International Conference on Robotics and Automation (ICRA 2007), Rome, Italy, pp. 927-933.

[5] Massa, B., Roccella, S., Carrozza, M. C. and Dario, P., 2002, "Design and development of an underactuated prosthetic hand," Proceedings of the IEEE International Conference on Robotics and Automation (ICRA 2002), Washington, DC, pp. 3374-3379.

[6] Townsend, W., 2000, "The BarrettHand grasper - programmably flexible part handling and assembly," Industrial Robot: An International Journal, 27(3), pp. 181-188.

[7] Krut, S., 2005, "A force-isotropic underactuated finger," Proceedings of the IEEE International Conference on Robotics and Automation (ICRA 2005), Barcelona, Spain, pp. 2325-2330.

[8] Dollar, A. M. and Howe, R. D., 2005, "Towards grasping in unstructured environments: grasper compliance and configuration optimization," Advanced Robotics, 19(5), pp. 523-543.

[9] Bégoc, V., Durand, C., Krut, S., Dombre, E. and Pierrot, F., 2006, "On the form closure capability of robotic underactuated hands," Proceedings of the IEEE International Conference on Control, Automation, Robotics and Vision (ICARCV'06), Singapore.

[10] Kragten, G. A. and Herder, J. L., 2010, "The ability of underactuated hands to grasp and hold objects," Mechanisms and Machine Theory, 45, pp. 408-425.

[11] Kragten, G. A. and Herder, J. L., 2010, "A platform for grasp performance assessment in compliant or underactuated hands," Journal of Mechanical Design, 132, p. 024502. 
[12] Krut, S., Bégoc, Dombre, E. and Pierrot, F., 2010, "Extension of the form-closure property to underactuated hands," IEEE Transactions on Robotics, 26(5), pp. 853-866.

[13] Dollar, A. M. and Howe, R. D., 2011, "Joint coupling design of underactuated hands for unstructured environments," International Journal of Robotics Research, 30(9), pp. 1157-1169.

[14] de Silva, C. W., 2009, Sensors and Actuators-Control System Instrumentation, Taylor \& Francis, CRC Press, Boca Raton, Fl.

[15] Fearing, R. S., 1986, "Simplified grasping and manipulation with dexterous robot hands," IEEE Journal of Robotics and Automation, 2(4), pp. 188-195.

[16] Saliba, M. A., 1991, "Kinematic, force and dexterity analyses of planar robotic grasping", M.A.Sc. thesis, University of British Columbia, Vancouver, Canada.

[17] Contensou, P., 1963, "Couplage entre frottement de glissement et frottement de pivotement dans la théorie de la toupee," in Ziegler, H. (Ed.) Kreiselprobleme und Gyrodynamics, Proceedings of the IUTAM Symposium, Celerina, 1962. Springer-Verlag, Berlin, pp. 201-216.

[18] Zhuravlev, V. G., 1998, "The model of dry friction in the problem of the rolling of rigid bodies," Journal of Applied Mathematics and Mechanics, 62(5), pp. 705-710. 